\title{
Evaluation of a Prognostic Decision Support System in a Neonatal Intensive Care Unit
}

\author{
by \\ Neda Faregh \\ B.A., Psychology, Carleton University 1998 \\ A thesis submitted to \\ the Faculty of Graduate Studies and Research \\ in partial fulfillment of the requirements for the degree of \\ Masters of Art \\ in Psychology \\ Department of Psychology \\ Carleton University, Ottawa, Ontario, Canada \\ (C) Neda Faregh, 2004 \\ The undersigned hereby recommend to \\ the Faculty of Graduate Studies and Research \\ acceptance of the thesis 1
}

Evaluating a Prognostic Decision Support System in a Neonatal Intensive Care Unit 


\author{
National Library \\ of Canada \\ Acquisitions and \\ Bibliographic Services \\ 395 Wellington Street \\ Ottawa ON K1A ON4 \\ Canada
}

Bibliothèque nationale

du Canada

Acquisisitons et services bibliographiques

395 , rue Wellington Ottawa ON K1A ON4 Canada
Your file Votre référence ISBN: 0-612-93897-2

Ourfile Notre référence ISBN: 0-612-93897-2
The author has granted a nonexclusive licence allowing the National Library of Canada to reproduce, loan, distribute or sell copies of this thesis in microform, paper or electronic formats.

The author retains ownership of the copyright in this thesis. Neither the thesis nor substantial extracts from it may be printed or otherwise reproduced without the author's permission.
L'auteur a accordé une licence non exclusive permettant à la Bibliothèque nationale du Canada de reproduire, prêter, distribuer ou vendre des copies de cette thèse sous la forme de microfiche/film, de reproduction sur papier ou sur format électronique.

L'auteur conserve la propriété du droit d'auteur qui protège cette thèse. $\mathrm{Ni}$ la thèse ni des extraits substantiels de celle-ci ne doivent être imprimés ou aturement reproduits sans son autorisation.
In compliance with the Canadian Privacy Act some supporting forms may have been removed from this dissertation.

While these forms may be included in the document page count, their removal does not represent any loss of content from the dissertation.
Conformément à la loi canadienne sur la protection de la vie privée, quelques formulaires secondaires ont été enlevés de ce manuscrit.

Bien que ces formulaires aient inclus dans la pagination, il n'y aura aucun contenu manquant. 
PAGINATION ERROR.

TEXY COMPLETE.
ERREUR DE PAGINATION.

LE TEXTE EST COMPLET. 


\author{
Abstract \\ Evaluation of a Prognostic Decision Support System in a Neonatal Intensive Care Unit \\ by Neda Faregh \\ Thesis Supervisor: Dr. Craig Leth-Steensen \\ Department of Psychology
}

\begin{abstract}
In order to evaluate the potential effectiveness of a prognostic Decision Support System in a Neonatal Intensive Care Unit, quantitative and qualitative analyses were undertaken. Seven Neonatologists were interviewed and a database of neonatal information was analyzed to compare two predictive models of neonatal mortality. The results indicated that neonatologists will likely welcome the use of a prognostic Decision Support System in their practices, particularly for counseling of neonatal patients' parents and potentially with patient management. The neonatologists were not completely satisfied with the use of the variables from the SNAPPE-II and MIRG models alone. Whereas these variables present a good starting point, they do not provide all of the required information needed for prognostic decisions. The interview results supported the notion that the use of a prognostic Decision Support System could potentially lead to problems arising from heuristic and memory biases. Furthermore, statistically driven rankings of the SNAPPE-II and the MIRG model variables did not correlate significantly with those provided by neonatologists. As well, the MIRG model had a significantly higher area under the Receiver Operating Characteristic Curve than the SNAPPE-II model, indicating that it does a better job of predicting outcome probabilities which leads to a more accurate classification of those outcomes.
\end{abstract}




\section{ACKNOWLEDGMENTS}

My appreciation goes to my thesis supervisor, Dr. Craig Leth-Steensen, for his direction and support. Many thanks to my thesis committee: Dr. Mary Gick, Dr. William Petrusic, and Dr. Robin Walker. I appreciate their feedback and help.

I acknowledge and thank Dr. Gitte Lindgaard for her help and direction at the early stages of this thesis work. I am grateful to Dr. Walker for facilitating interviews with Neonatologists. My thanks go to Dr. Monique Frize, for providing access to a sample of the Canadian Neonatal Network Database. 


\section{TABLE OF CONTENTS}

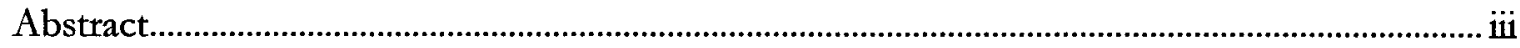

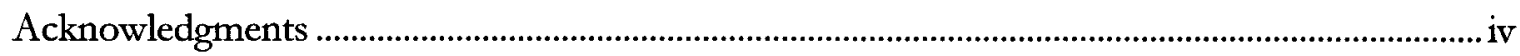

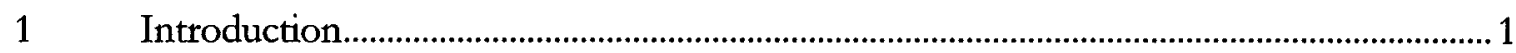

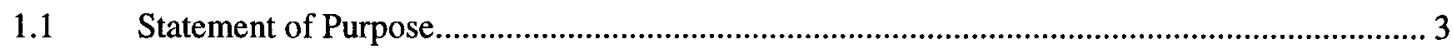

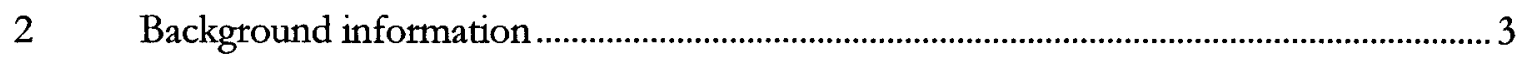

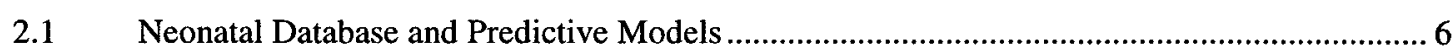

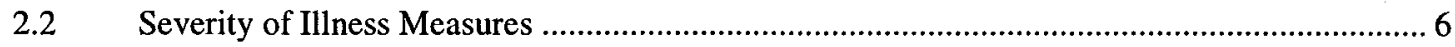

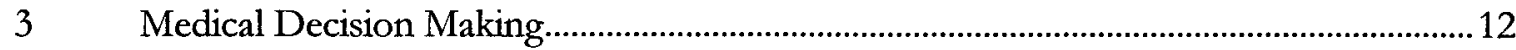

$4 \quad$ Normative Approach in Clinical Decision Making.......................................................14

4.1 Heuristics and Biases and Their Role in Medical Judgements................................................ 19

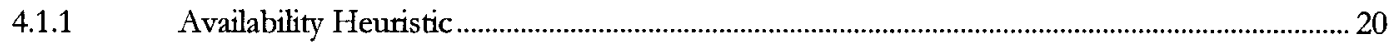

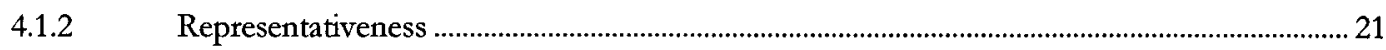

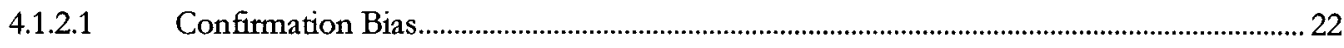

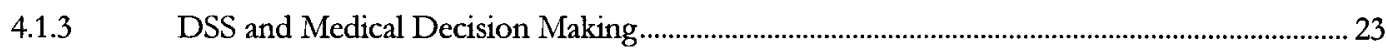

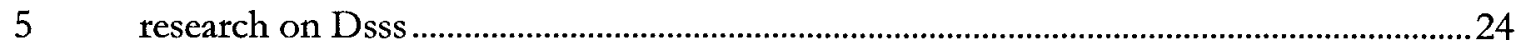

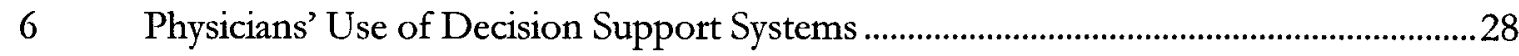

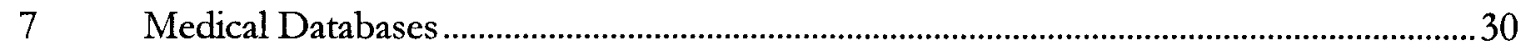

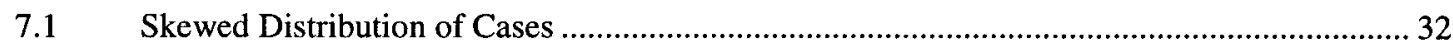

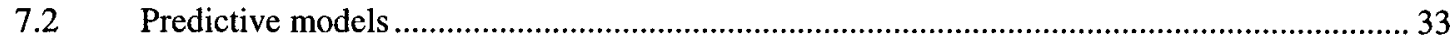

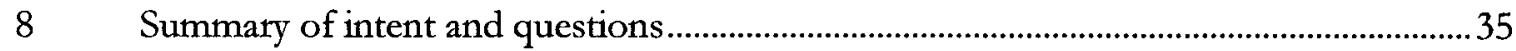

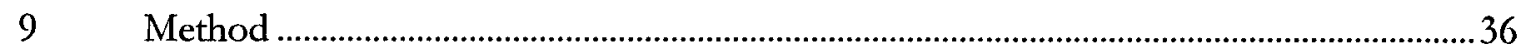

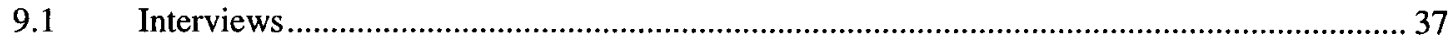

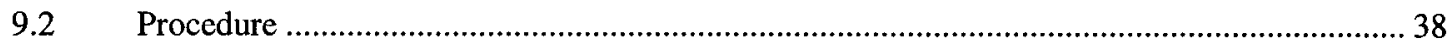

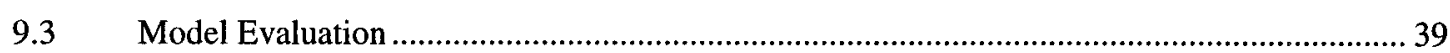

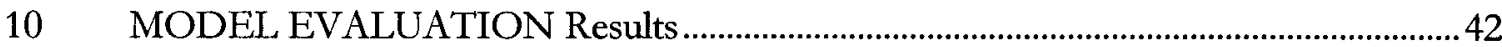

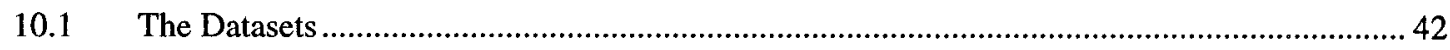

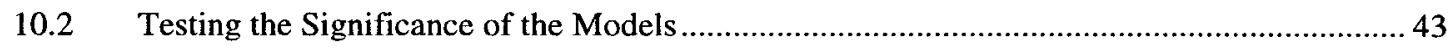

10.2.1 Approach 1: Assessing Significance of Variables in the Models as a Whole ...............................4 44

10.2.1.1 Model Comparison ..................................................................................................................... 46

10.2.1.2 Comparing the Models with Low Birth Weight Cases Only ..................................................... 47

10.2.2 Approach 2: Assessing the Significance of the Variables in the Model Individually ................ 48 
10.2.2.1 Relative Importance of the Variables in Each Model .......................................................5 50

10.2.2.2 Interactions Among Model Variables .............................................................................. 52

10.2.2.3 A Closer Look at an Interaction Variable ............................................................................ 54

10.3 Examining the Predictiveness of the Models ..................................................................55

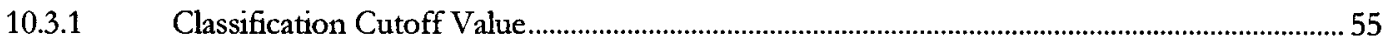

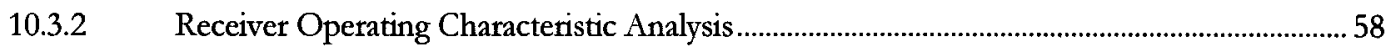

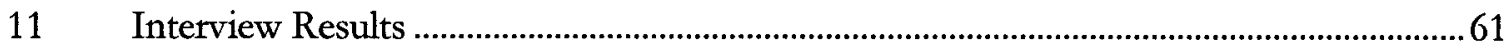

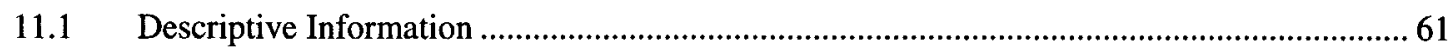

11.2 General Views About the Value of a Prognostic DSS in a NICU.....................................63

11.3 Views About the Accuracy, Reliability and Trustworthiness of DSS Output ......................66

11.4 Opinions About the Effects of Information From a DSS on Participants' Confidence...........69

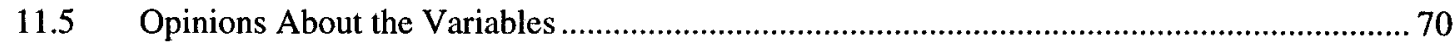

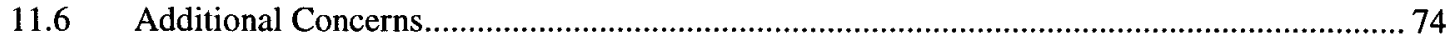

11.6.1 Concerns Regarding Lack of Trend Information ............................................................... 74

11.6.2 Concerns Regarding Lack of Gestation Age as a Variable ................................................... 74

11.6.3 Concerns About Dated Information and the Lack of Centre-Specific Data ......................... 74

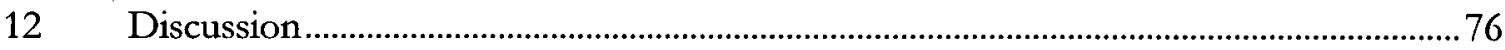

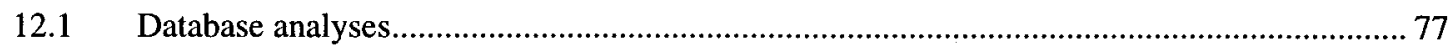

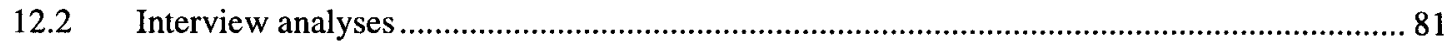

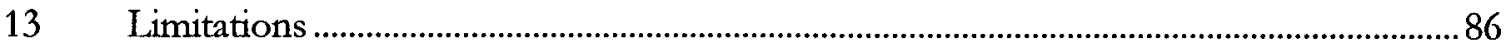

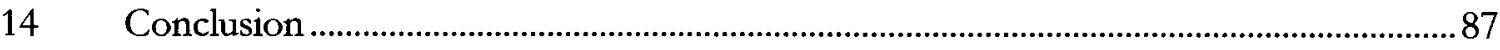

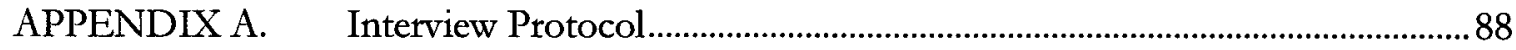

APPENDIX B. Full Correlation Matrix ............................................................................95

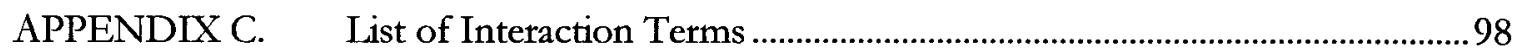




\section{LIST OF TABLES}

Table 1. Variables Used by SNAP, SNAP-II and SNAPPE-II ................................................ 8

Table 2. Effects of Using Decision Support on Correct Diagnosis in \% ...............................27

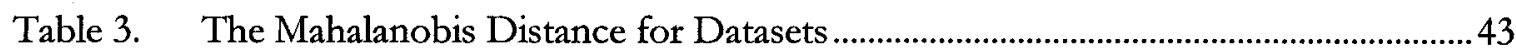

Table 4. Database Summary Information with Outliers Removed..........................................43

Table 5. Omnibus Tests of Model Coefficients in the Different Datasets .............................45

Table 6. Omnibus Tests of Model Coefficients for Different Models Using the Composite Dataset.

Table 7. Measures of Association between the Predictors and the Outcome for the

Different Datasets.

Table 8. Comparison of the Model Chi-squares for the SNAPPE-II model and the Composite Model

Table 9. Comparison of the Model Chi-squares for the MIRG model and the Composite Model.

Table 10. Comparing the SNAPPE-II and the Composite Models for Low Birth Weight Data Only.

Table 11. Comparing the MIRG and the Composite Models for Low Birth Weight Data Only......

Table 12. Individual Tests of the Significance of the Variables in Models using the Composite Dataset

Table 13. Odds Ratios in Standardized Units for All Variables in Each of the Models in the Composite Dataset.

Table 14. Cutoff Values and Associated Percent Predictions for SNAPPE-II variables in Composite Dataset.

Table 15. Comparison of the Areas Under the ROC Curve for Models With and Without their Interaction Terms.

Table 16. Participants' Reasons for Computer Use .62

Table 17. Participants' Rankings for Accuracy, Reliability, and Trust of a Prognostic DSS Output

Table 18. The Three Most Important Variables for Prognosis According to Participants.. 71

Table 19. Ranking Statistics For the 15 MIRG and SNAPPE-II Variables (Where the Most Important Rank = 1) 
Table 20. Spearman Correlation Coefficients for the 15 Composite Variable-Rankings by

Clinicians, Simple Regressions, and Partial Regressions. .....................................................73 


\section{LIST OF FIGURES}

Figure 1. Estimated Marginal Means of Predicted Probability for Lowest Blood Pressure as a Function of Lowest Temperature in 3 Categories in Ascending Order. . .55

Figure 2. ROC Curve for Discriminative Ability of MIRG vs. the SNAPPE-II Model . .60 
Evaluating a Prognostic Decision Support System in a Neonatal Intensive Care Unit

$$
\text { By Neda Faregh }
$$

\section{INTRODUCTION}

In clinical medicine, the accuracy of prognostication is an important issue as it influences clinicians' decisions involving the course of therapy for patients. In neonatal medicine, there is often a degree of ambiguity surrounding the likelihood of survival for some neonates admitted to a Neonatal Intensive Care Unit (NICU). Increasing prognosis accuracy is an important goal of clinical medicine as it can have a direct influence on the appropriateness of selected therapy. Given the fact that neonatologists often overestimate the probability of survival in their patients including moribund neonates, a more accurate prognosis allows neonatologists to communicate a more realistic set of options and outcomes to parents of NICU patients during family counseling. Likewise, this reduces the subjection of the family to false hopes and further tension throughout a process that is already very difficult. The decision regarding what therapy is to be attempted is best made with a more accurate perception of true outcome and can result in a reduction of intensive, distressing, and unnecessary treatment of patients who will not survive. It will also reduce needless use of hospital resources and increases in health care costs. In an attempt to improve prognostic decisions, medical Decision Support Systems (DSS) intended to support prognostic decisions regarding the survival of neonates have been developed.

In order to support prognostication, a prognostic DSS requires predictive models and large repositories of pertinent information. Prognostic models are tools for helping decision making that combine specific components of patient data to predict clinical outcomes. 
Pertinent information is retrieved from large patient databases. Information retrieval algorithms, also called data mining or knowledge discovery, allow for detection of relationships between different elements of information, post hoc, and recognition of patterns which are subsequently tested statistically. Health care is one area where data mining is frequently applied, particularly to support medical decision making. Data mining is often used in conjunction with medical DSSs.

Case based reasoning is a method of retrieving information for knowledge based systems including medical DSS. It is also believed to be a method of human decision making. Some authors argue that decision makers recall situations or problems similar to the one at hand and apply the solution of a similar former problem to the new problem (Schmidt, Montani, Bellazi, Portinale, \& Gierl, 2001). The assumption is that similar problems can be solved by similar solutions. When the case based reasoning technique is used by a computer system, that system is called a Case Based Reasoner (CBR). A CBR retrieves information from a database by searching for the most similar cases to some particular case.

In recent years, a of number DSSs, including CBR-based DSSs have been developed to support medical decision making, but the nature and extent of their usefulness in clinical settings remains unknown. This is because few such systems are incorporated in clinical use and few studies have examined how these systems are being employed. Some studies have concluded that the use of medical DSSs in clinical settings, for example, radiology, pharmacology, psychiatry, and oncology, can be beneficial (Lisboa, 2002; Schmidt et al., 2001). Others point out the reluctance of clinicians to adopt these systems Johnston, Langton, Haynes, \& Mathieu A., 1994; Lisboa, 2002). This thesis investigates how a DSS intended to 
support prognostic decision making in a NICU may be received by neonatologists. A CBRbased medical DSS needs to rely on medical data often originating from hospital databases which contain an array of information for several thousand patients, however only a few select information categories are used for matching purposes by the DSS. These categories are determined by predictive models which provide the specific variables used for information retrieval. The perception of a DSS by neonatologists depends on many factors including its potential use, such as the purposes for which the DSS might be employed, and its underlying data. A DSS needs to be reliable and operate with a clinically credible prognostic model. This thesis examines neonatologists' perception of medical databases and the data as well as predictive models used by a DSS to provide prognostic support.

\subsection{Statement of Purpose}

This thesis examines how a medical DSS intended to support prognostic decision making in a NICU may be received by its intended users. It investigates neonatologists' perception of a CBR based medical DSS and its underlying databases. In addition, the thesis analyses the prognostic ability of two predictive models of neonatal mortality, as prognosticating models to be used by the DSS. The statistical predictive abilities of these models are then compared with neonatologists' judgment of their prognosticity.

\section{BACKGROUND INFORMATION}

Before further discussion of DSS and clinicians' views, some background knowledge of the prognostic DSS of concern in this thesis will be helpful to the reader. This section presents information about the origin of this CBR-based DSS, the data it uses, and how it came to be. 
The Medical Intelligence Research Group (MIRG) is part of the Artificial Intelligence and Biomedical Instrumentation Laboratory of Carleton University. The group consists of engineering faculty and students as well as physicians in the Ottawa area. The focus of this group is to apply CBR and Artificial Neural Network (ANN) techniques to medical databases in order to develop medical decision aids. The group has developed a CBR system for an adult Intensive Care Unit (ICU) and has adapted it for use in NICUs by employing neonatal data. The CBR allows users to query a database by entering data about a particular patient they are treating in order to retrieve information about similar patients. A common difficulty faced by all applications using databases is the issue of missing values. Values may be missing because either they were not needed, or not available, or overlooked and not collected. In a medical database, values may be missing because certain laboratory test results were not needed for a patient, or were lost, or for other reasons, such as time limits, were not recorded. Because medical databases contain many missing values, ANN's have been incorporated into the CBR to make an ANN and CBR hybrid (Ennett, 2003). This hybrid retrieves the 10 nearest matches using a nearest-neighbour algorithm and imputes missing values with the mean of these 10 matched cases. The output is rank ordered according to the similarity of cases to the data the user has entered, and generally is presented in a spreadsheet-type screen output. At the time of this writing, the ANN-CBR hybrid is not fully developed, therefore information about the exact display of the output is not available. It will potentially display information as cases (patients) retrieved from the database. Each case would consist of a number of physiological variables such as birth-weight and body temperature, and the outcome (lived or died). The output would be presented in a spread-sheet format with 10 rows for 10 patients and the 
variables in columns. Further information about the output and its uses will be discussed in a later section.

In order to obtain similarities and match cases, the CBR needs to know which variables to match on. In addition, the CBR needs to assign weights (i.e., importance) to each variable (note that the variable values used by the CBR are first normalized). The variables are suggested by a model, in this case a neonatal mortality predictive model. The weights may be unit weights where all variables are given a weight of 1 , or they may be clinician-assigned weights where the user can decide the importance of each variable. Alternatively, weights maybe assigned by a linear $\mathrm{ANN}$ which has been trained to associate the variables as inputs with the outcomes as outputs. The ANN-CBR Hybrid system uses weights obtained by a linear ANN. These weights reflect the importance placed on each variable with respect to their influence on the outcomes. Using these weights, the CBR employs its matching algorithm to select the most similar cases. Ennett (2003) reports that an established neonatal predictive model with imputed missing values obtained using the CBR with ANN weights, discriminated outcomes as well as the same model with imputed missing values obtained using the CBR with more "clinician-friendly" weight assignments. In addition, using a linear ANN, Ennett developed a new predictive model for neonatal outcome prediction (the MIRG model). Further details of the MIRG model and its variables will be discussed in the next section. This thesis compares the predictive ability of the MIRG model with an already established predictive neonatal model, using statistical methods. It also examines the weightings of the variables derived by these statistical methods and compares them with weights derived statistically and with weights suggested by the subject matter experts (neonatologists). 


\subsection{Neonatal Database and Predictive Models}

The MIRG used the medical database of the Canadian NICU Network (2003) to test the ANN-CBR hybrid and to develop a new neonatal predictive model. This network consists of a group of researchers who collaborate on research issues relating to NICUs in Canada. The data populating the neonatal database were collected over a 22-month period (1996-1997). The NICU patient admission records were collected by 17 participating hospitals across Canada. This cooperation led to a database containing information for 20,488 admissions to NICUs during this period. The admission records have generated some 200 categories of information including physiological measures and administrative data such as patient addresses. Only some of these information categories are used by the ANN-CBR hybrid. Only the variables that are part of the predictive model used by the system are selected and employed. One established model, developed by the Canadian NICU Network, and used by the ANN-CBR hybrid is a measure of the neonatal severity of Illness. The MIRG model is based on and derived from this measure of illness severity.

\subsection{Severity of Illness Measures}

Prognosis of patient outcome such as neonatal mortality is important because it facilitates and affects patient and resource management. According to Civetta (1997), because clinical approaches to decision making remain imperfect, a number of methods have been devised as quantitative indices to afford a more accurate tool for evaluation of the severity of illness and mortality probabilities. Predicting the probability of survival is important for clinical decision making as it can affect clinicians' choice of therapy. For example, a severely ill neonate with no or a minute chance of survival may only be subjected to what is termed "comfort care", whereas an infant with better survival chances might receive more intensive therapy. 
Other important factors concerning accurate prediction of survival include resource allocation and cost-containment efforts (Civetta, 1997). While these are essential to an ICU operation, they implicate a gamut of political and ethical factors regarding the choice of therapy that are beyond the scope of this thesis. No issues pertaining to therapeutic and economic aspects of patient care will be addressed here. The main issue considered in this thesis is survival prediction.

One project conducted by the Canadian NICU Network examined how differences in clinical practice among Canadian NICUs affect outcomes and how the availability of resources needed to provide the required care may affect quality of care. Outcome is defined as inhospital mortality and survival of sick newborn infants. Through this project, the Canadian NICU Network has developed a mortality risk model for NICU patients based on the severity of illness. This mortality risk model is called the "Score for Neonatal Acute Physiology" (SNAP). The SNAP index was developed in 1993 and relied exclusively on physiological measurements such as vital signs (e.g., lowest mean blood pressure) and laboratory test results (e.g., white blood cell count) regardless of diagnosis or therapy. Its aim was to provide clinicians with information above the traditionally available neonatal risk factors such as extreme birth weight, gestational age, sex, race, and Apgar scores (Richardson, Gray, McCormick, Workman, \& Goldmann, 1993). These measures were chosen through consultation with a team of neonatologists, pediatrics, and nurses. The SNAP scoring system uses 37 physiological variables scoring the worst physiologic problems in each organ system during the first 24 hours after admission to NICU. The SNAP score is independent of diagnosis or therapy as it reflects only the degree of physiological derangement in organ systems (Richardson et al., 1993). SNAP was later simplified to be a less "cumbersome" and 
more "parsimonious" scoring system (Richardson, Corcoran, Escabar, \& Lee, 2001) by reducing the number of variables from 37 to 6 and also reducing the time frame for scoring to the first 12 hours after admission. The simplified version is called the "Score for Neonatal Acute Physiology II" (SNAP-II). The 6 variables were arrived at by eliminating variables from the SNAP that had non-significant statistical correlations to mortality, were used infrequently, or were deemed unreliable "as assessed by feedback from abstractors[those responsible for abstracting data]"(Richardson et al., 2001).

However, the SNAP-II does not include perinatal risk factors. Perinatal risk factors are birth weight, information about whether the baby is small for its gestational age, and Apgar scores (see Table 1 for a description of Apgar scores). Hence, in order to make the SNAP-II useable in neonatal setting, these 3 perinatal risk factors were added to SNAP-II, yielding the "Score for Neonatal Acute Physiology II with Perinatal Extension" (SNAPPE-II). The SNAPPE-II uses 9 physiological variables to predict mortality. The addition of the 3 perinatal risk variables was believed to give the new scoring system, SNAPPE-II, more predictive power, in part, the authors argued, because each of these variables on their own are good predictors of mortality (Richardson et al., 1993; Richardson et al., 2001). See Table 1 for a list of variables included in the SNAP, SNAP-II, and the SNAPPE-II indices.

Table 1. Variables Used by SNAP, SNAP-II and SNAPPE-II

\begin{tabular}{|c|c|c|}
\hline SNAP Variables & SNAP-II & SNAPPE-II Variables \\
\hline 1. Mean Blood Pressure Low & $\begin{array}{l}\text { 1. Lowest Mean Blood } \\
\text { Pressure (bloodp) }\end{array}$ & $\begin{array}{l}\text { 1. Lowest Mean Blood } \\
\text { Pressure (lbloodp) }\end{array}$ \\
\hline 2. Mean Blood Pressure High & & - \\
\hline 3. Temperature, Degrees & 2. Lowest Temperature in & 2. Lowest Temperature in \\
\hline
\end{tabular}


Fahrenheit Degrees Fahrenheit (ltemp) Degrees Fahrenheit (ltemp)

4. Ratio of Partial Pressure of Oxygen vs. Fraction of Inspired Oxygen (PO2/FiO2)

3. Ratio of Partial Pressure of 3. Ratio of Partial Pressure of Oxygen vs. Fraction of Oxygen vs. Fraction of Inspired Oxygen (PO2/FiO2) Inspired Oxygen (PO2/FiO2)

5. Urine Output, $\mathrm{mL} / \mathrm{kg} / \mathrm{h}$

4. Lowest Urine Output (lurine), $\mathrm{mL} / \mathrm{kg} / \mathrm{h}$

5. Lowest Serum pH (lserum) 5. Lowest Serum pH (lserum)

7. Seizure

6. Presence of Single or Multiple Seizures (seizure)
4. Lowest Urine Output (lurine), $\mathrm{mL} / \mathrm{kg} / \mathrm{h}$

6. Presence of Single or Multiple Seizures (seizure)

7. Birth Weight (Birthwt)

8. Small for Gestation Age (SGA)

9. Apgar Score at 5 Minutes (Apgar 5) $^{*}$

8. $\mathrm{Po}_{2}, \mathrm{~mm} \mathrm{hg}$

9. Heart Rate Low

10. Heart Rate High

11. Respiratory Rate

12. $\mathrm{PcO}_{2}, \mathrm{~mm} \mathrm{hg}$

13. Oxygenation Index

14. Hematocrit Low, $\%$

15. Hematocrit High, \%

16. White Blood Cell Count

\footnotetext{
* The Apgar score is taken 5 minutes after the birth of the baby. The Apgar score (an abbreviation for Activity, Pulse, Grimace, Appearance, and Respiration) is a number arrived at by scoring the heart rate, respiratory effort, muscle tone, skin color, and responsiveness of the newborn. The highest Apgar score assigned to a healthy baby is 10 , the lowest Apgar score assigned to a baby with no vital signs is 0 .
} 
17. Immature Total Ratio

18. Absolute Neutrophil Count

19. Blood Urea Nitrogen, $\mathrm{mg} / \mathrm{dL}$

20. Creatinine, $\mathrm{mg} / \mathrm{dL}$

21. Indirect bilirubin Low (by birth weight)

22. Indirect bilirubin High (by birth weight)

23. Direct bilirubin, $\mathrm{mg} / \mathrm{dL}$

24. Sodium, Low $\mathrm{mEq} / \mathrm{L}$

25. Sodium, High mEq/L

26. Potassium Low, mEq/L

27. Potassium High, $\mathrm{mEq} / \mathrm{L}$

28. Calcium (ionized) ${ }^{2}$ Low, $\mathrm{mg} / \mathrm{dL}$

29. Calcium (ionized) ${ }^{2}$ High, $\mathrm{mg} / \mathrm{dL}$

30. Calcium (total) ${ }^{2}$ Low, $\mathrm{mg} / \mathrm{dL}$

31. Calcium (total) ${ }^{2}$ High, $\mathrm{mg} / \mathrm{dL}$

32. Glucose (or reagent strip) Low $\mathrm{mg} / \mathrm{dL}$

33. Glucose (or reagent strip) High mg/dL.

34. Serum Bicarbonate Low, $\mathrm{mEq} / \mathrm{L}$ 
35. Serum Bicarbonate High, $\mathrm{mEq} / \mathrm{L}$

36. Apnea

37. Stool Guaiac

The SNAP indices are arrived at by assigning points, from a scale of 0 to 5 , to each variable based on the degree of severity of the value. The SNAPPE-II indices are arrived at in a similar fashion but with a slightly different point scoring scheme. The final index represents the degree of illness severity. The lower the score, the healthier is the baby. The SNAP and SNAPPE-II indices were validated prospectively at hospital NICUs and shown to be predictive of neonatal mortality. Note that points assigned to the SNAP and SNAPPE-II variables for calculating the SNAP and SNAPPE-II indices are independent of variable weights assigned to these variables when used in the ANN-CBR hybrid discussed earlier, and will also not be relevant to any statistical analysis performed in this thesis. The method of index calculation is discussed here only to describe these severity of illness measures and how they are used in practice.

The 9 variables in the SNAPPE-II index are the same ones used by the CBR to find matching cases and are the variables reported for matched cases. Again, it should be noted that the final SNAPPE-II score or index is not used by the DSS. The SNAPPE-II variables only, were initially chosen by the MIRG to use in the CBR system because its primary purpose is to help clinicians predict patient mortality and these variables were deemed to be useful for such a purpose. However, the MIRG has recently proposed a new set of variables (the MIRG model) as a neonatal mortality predictive model. The reasons for needing a new neonatal mortality model were that "although SNAP-II has proven clinically useful for a variety of 
outcomes, the final list of variables that predict important outcomes such as mortality is somewhat in question. This fact is important for a model's integration into clinical practice and acceptance by clinicians and other health care professionals [page 73]" (Ennett, 2003). The MIRG model consists of 13 variables, 7 of which are part of the SNAPPE-II model, plus 6 new variables. Based on ANN analyses, the two variables seizure and lowest mean arterial blood pressure that were part of the SNAPPE-II were considered as not contributing significantly to neonatal mortality outcome prediction by the ANN and hence, were removed. These variables were replaced by lowest platelet count, highest mean arterial blood pressure, highest respiratory rate, highest $\mathrm{PCO}_{2}$, highest sodium, and lowest glucose which were shown by the ANN to contribute significantly to neonatal mortality outcome prediction. The MIRG model and the selection of its variables will be discussed in more detail later.

\section{MEDICAL DECISION MAKING}

Medical decisions are mostly made under uncertainty. Elements of decision making common to all fields of medicine are prognosis, diagnosis, and interpretations of test results. These elements are often referred to as the "art of medicine" (Schwartz \& Griffin, 1986). The idea of medicine being practiced as an art conveys the importance of the use of intuition in what is held to be a scientific field of rules and facts. Nevertheless, medical decisions are almost always based on some kind of probability estimates that inform the clinician's decisions about patient treatment.

In a classical study, Meehl (1954) reviewed 20 studies in which mathematical equations, that is actuarial or linear models, were compared to clinical judgements. Linear models use declarative knowledge and rational analysis to decompose information into small units of basic 
elements. They also allow for de-contextualization of these units and for simple calculations (Klein, 1998). In all the studies reviewed by Meehl, the results showed that the actuarial models either did as well as or outperformed the experts (Meehl, 1954).

Since Meehl's publication, many researchers have reported similar findings that predictions by actuarial models are more reliable than predictions by human experts. For example, actuarial models have been more reliable than clinicians in the case of the Minnesota Multiphasic Personality Inventory (MMPI) profile for diagnosing patients as either neurotic or psychotic (Goldberg, 1968). In other fields, actuarial models have outperformed admission officers in predicting academic performance of students in medical schools (De Vaul et al., 1957), law schools (Swets, Dawes, \& Monohan, 2000), and graduate school in psychology (Dawes, 1971).

In general though, the research on the use of such models has shown that despite the debates and the evidence, clinicians and patients have been reluctant to embrace medical actuarial models and have not abandoned the clinical approach which relies heavily on intuitive judgement and prior experience. This may be due to the fact that although one advantage of linear models is in their allowing for an orderly and systematic approach to analysis, and although they can outperform experts, their use is not always advisable. There are many situations where these models are not "proper" linear models (Dawes \& Kagan, 1988). An "improper" linear model is one in which the weights assigned to different criteria are not chosen to optimize the relationship between the variables and the outcome. The importance placed on each variable may be based on subjective information. There are many situations where continuous and iterative expert judgement is required and "continued application of the 
model neglects any new conditions that may have a bearing on the judgement [page 117]" (Ebert \& Kruse, 1978). Dawes (1979) states that no clinical judgment can prove superior to a statistical prediction model as long as both predictions are based on codable input variables. However, Dawes suggests that it is the experts who can best choose and code the most appropriate variables. But, the integration of the information is best done by statistical models (Dawes, 2001). The implication for a DSS is that the system can successfully integrate and compute the results for a particular problem but the system must first be given the appropriate model.

\section{NORMATIVE APPROACH IN CLINICAL DECISION MAKING}

Generally, to make decisions about a patient's status of health, physicians should use a normative approach to decision making by considering probabilities and utilities when making clinical judgements, where probabilities and utilities are multiplied and the option with the highest utility is chosen. Expected utility theory (Von Neuman \& Morgenstern, 1947) is a normative theory prescribing the requirements of rational decision making. The theory calls for comparison of alternatives according to objective probabilities and choosing the strategy that yields the best outcome. Not all decision makers maximize their utilities according to this theory because they fail to follow the prescriptive axioms, and because the best outcome is not easily or unequivocally determined. An extension to the expected utility theory was developed to allow for the subjective probabilities of outcomes (Savage, 1954). Subjective probability is the degree of certainty one can assign to an event without objective information. The subjective probability of an event could be based, for example, on past experiences. Savage's theory is called subjective expected utility theory and includes a person's subjective 
probabilities about the occurrence of an outcome when objective probabilities are unknown (Plous, 1993).

In medicine, utilities include intangible variables such as pain and quality of life. For example, the utility of false positive test results vary considerably from false negative results. Treating a healthy patient suspected of having a disease has a utility that is very different from that of not treating a patient who might have the disease. In addition, utilities may change depending on the probability of survival and the anticipated end result of treatment. In practice, it is not clear when or how a "small chance" of survival is attributed to a patient. The chance of survival for a NICU patient obviously depends on the state of a baby's health and other relevant variables such as the birth history or the mother's health. It also depends on the clinicians' ability to treat the problem, as well as the ability of the nurses, the NICU facilities, and the existing nursing or clinician workload to name but a few covariates. The potential long term outcome is another important factor considered by the clinical decision makers. The quality of life after survival may be as important as the possibility of survival. These covariates also affect the overall expected utility of the prognostic decision. Therefore, the "best" decision is often subjective and contingent on many different circumstances surrounding each case.

The most difficult tasks clinicians face is deciding on the "best" choice for achieving the highest utility. When a model's predictive ability is evaluated, the utility assigned to falsenegative and false-positive results must be taken into consideration. This utility can be thought of as the cost of error. Among the information available to clinicians regarding a patient are patient history, tests, and examination results which need to be understood in terms of the 
probability that they lead to an accurate diagnosis. Test results are not always reliable or correct. The probability of a patient's health status depends on the patient's history, age, and race etc. The objective probability of test results being correct is available to clinicians but the judgment of a patient's health status is a subjective evaluation. It depends on how accurately the physician can predict the true condition of the patient from the available information sources.

An interpretation of objective and subjective probabilities is necessary to make decisions about a patient's prognosis or treatment. However, most clinicians have difficulty calculating the probability of a particular outcome given the evidence. This probability is known as the posterior probability and can be calculated using the Bayes' theorem (Eddy, 1982). But not all clinicians receive sufficient instruction in statistics or statistical arguments. The result of a survey of physicians' statistical training in medical schools is worth noting here. A survey of medical schools showed that the typical physician is required to study at most one statistics course as part of their medical training. The average number of training courses received at the undergraduate and medical schools as well as continuing education was 1.35 . The time since a most recent course averaged 18 years (Klatzky, Geiwitz, \& Fischer, 1994).

The difficulties physicians face with regards to probabilistic reasoning is a well researched problem. For a detailed example, see Eddy's study (1982) which investigated clinicians decisions on whether patients with a suspicious breast mass needed a breast biopsy.

The application of the Bayes' theorem is as follows:

$\mathrm{P}($ disease $\mid$ evidence $)=\mathrm{P}($ evidence $\mid$ disease $) \mathrm{P}($ disease $) / \mathrm{P}($ evidence $/$ disease $) \mathrm{P}($ disease $)+\mathrm{P}($ evidence $\mid$ no disease) $\mathrm{P}$ (no disease) 
Where $\mathrm{P}$ (disease / evidence) is the probability of the patient having the disease given that the evidence is positive, also called the posterior probability;

$\mathrm{P}$ (evidence $\mid$ disease) is the probability of correct diagnosis if the patient has the outcome, the sensitivity or the true positive rate;

$\mathrm{P}$ (disease) is the probability that the patient has the outcome determined by the physician's subjective judgement of the patient's health status and characteristics such as age and family history, also called the prior probability;

$\mathrm{P}$ (evidence/no disease) is the probability that the evidence is positive given that the outcome is actually not found, the false positive rate or 1- specificity;

$\mathrm{P}$ (no disease), is the probability that the patient's hypothesized outcome is not present (or that the outcome does not take place). This is the complement of $\mathrm{P}$ (disease) and therefore the complement of the base-rate where $\mathrm{P}($ disease $)+\mathrm{P}($ no disease $)=1$.

Sensitivity and specificity rates associated with information about the accuracy of test results, that is, the potential for true positives and false positives associated with each test, are available in the medical literature. The subjective probability, $\mathrm{P}$ (disease) and therefore $\mathrm{P}$ (no disease), is to be determined a priori by the physician. If the reference group is precisely known, this probability can be found objectively. However, in reality this probability is estimated subjectively by the physician. Eddy (1982) reports that about $95 \%$ of physicians misinterpret statements about posterior probabilities, $\mathrm{P}$ (disease $\mid$ evidence), and sensitivity, that is, the true positive rate or $\mathrm{P}$ (evidence/disease). They often confuse the two. This confusion often leads to gross overestimation of the probability of the outcome in a patient. Similar 
findings have been reported by others (Einhorn \& Hogarth, 1982; Einhorn \& Hogarth, 1983; Schwartz, Kassirer, \& Essig, 1973). The problem persists even in the case of physicians specifically trained to overcome it, though training has proved somewhat helpful (BeythMarom \& Fischhoff, 1983). Overall, it seems that physicians, like lay people, are not effective in calculating probabilities and interpreting them correctly. Some argue that confusion involved in probabilistic reasoning is due to difficulties in understanding conditional probabilities, but even those trained in statistics and Bayes' theorem cannot always overcome the confusion. It is argued that failure to correctly apply Bayes' theorem is simply due to cognitive deficits inherent in human judgement (Tversky \& Kahneman, 1973).

A major benefit of a prognostic DSS relying on a historical patient database is that the outcomes associated with its outputs are known. However, the output given to the physician in the form of 10 similar cases complete with outcomes, does not offer the clinicians information about any of the probabilities discussed above. A DSS could potentially present the clinicians with posterior probabilities, but the DSS discussed in this work cannot distinguish between the different cases in the database in terms of the underlying disease, nor does it know the prior probability of outcome for each case. One possible scenario of clinician-DSS interaction is that the clinician assigns a subjective prior probability of survival to the patient. When consulting the DSS, the physician is presented with information regarding the outcome of 10 similar cases. The ratio of survivors to non-survivors can then be easily calculated. The proponents of the CBR-based DSS hope that this new probability will influence, and possibly encourage revision of, the physician's original opinion about the probability of the patient's outcome. 


\subsection{Heuristics and Biases and Their Role in Medical Judgements}

Human judgment is fraught with cognitive deficits which have been studied in the past several decades. Simon (1956) introduced the idea of bounded rationality as another way of explaining the limitations to human cognition in complex judgment. He suggested that people use "simplified problem representations" allowing them to reduce cognitive load and make reasonably accurate judgements in complex situations. He called these "judgement heuristics", used as rules of thumb to help in making decisions. In general, judgement heuristics serve decision makers well and result in correct decisions but there are many instances where they fail (Simon, 1956). Since the mid 1970's, a great deal of research has described these judgement heuristics and have depicted circumstances where these heuristics lead to errors or what is termed "cognitive biases" (Bell, Raiffa, \& Tversky, 1988; Fischhof, Slovic, \& Linchtenstein, 1977; Fischhof, 1977; Fischhof, 1982; Gilovich, Griffin, \& Kahneman, 2002; Kahneman, 1973; Tversky \& Kahneman, 2001)

A heuristic is a rule of thumb used to process information quickly. It is defined as a strategy to reach decisions without having to consider all possible options (Gigerenzer \& Todd, 1999). Heurists are shortcuts that simplify complex decision tasks and most often produce correct or almost correct results. There are, however, a number of well studied and predictable biases that can result from using heuristics. Two broad classes of heuristics involved in probability judgments are discussed in this section. They are the availability, and the representativeness heuristics. 


\subsubsection{Availability Heuristic}

The availability heuristic occurs when the probability of the occurrence of an event is judged based on the ease with which instances of that event are remembered (Tversky \& Kahneman, 1973) rather than from a consideration of all possible cases. The ease with which an event is remembered may be due to familiarity or salience. More recent occurrences are better remembered, or more available, than earlier ones. The availability heuristics can lead to biases in medical decision making. Rarity of a disease, vividness of a particular patient's case, its consequences for the physician or the patient, for example, can cause a clinician to overestimate the probability of an unusual disease (Sox, Blatt, Higgins, \& Marton, 1988; Sox et al., 1988).

Normally, the availability heuristic leads to accurate judgements because events that are remembered easier are those that occur more often. However, this isn't always the case. A study on the subjective mortality estimates and prediction (Christensen-Szalanski, Beck, Christensen-Szalanski, \& Koepsell, 1983) showed that doctors asked to estimate mortality rates of different diseases estimated the diseases with most journal coverage as more fatal. These authors also found a high correlation between coverage of a particular disease in medical journals, and the doctors' estimates of its mortality rates. In addition, they found that the number of patients doctors had diagnosed with a specific disease had a higher correlation with the physician's estimates of mortality rates of the disease. This is in accord with earlier research by Trersky and Kahneman (1973) showing that more available information is judged to be more frequent, even when it is not. 
The assumption underlying the CBR is that retrieving cases that are similar to a patient at hand helps by making a more realistic clinical picture available to the physician. If the CBR output showing the 10 most similarly matched cases contains information about matched patients who survived, the physician is likely to estimate the probability of mortality according to these outcomes. Conversely, if the similarity-matched patients had died, the clinician is more likely to base her judgement on this outcome since it happens to be the available, recent, and perhaps salient clinical picture. As long as the clinical picture is painted correctly, the CBR can provide objective information and can potentially reduce at least some biases that occur due to availability heuristics.

\subsubsection{Representativeness}

The representativeness heuristic is often relied on when one must estimate the probability that one event will lead to another. According to Kahneman and Tversky (1982) the likelihood that event $A$ will lead to event $B$ is assessed by the extent to which $A$ is representative of, or resembles, B. Their research has shown that people judge probabilities the same way that they judge similarities. For example, they asked a group of subjects to judge the probability that a helpful, quiet, tidy, and shy man is a salesman, a librarian, a physician, or a farmer. Subjects assessed the probability of the man's occupation based on how the character descriptions were representative of, or similar to, a given occupation's stereotype. That is, the probability that the man was a librarian was determined based on how his characteristics were representative of a stereotypical librarian. This approach could lead to errors if the man in question lived in a large community of farmers and few librarians. Another example also

reported by Tversky and Kahneman (1973) describes judging the majors of a group of students described as nerds. Most people would assign a high probability to the students being 
science majors, regardless of how common such majors were. To avoid the error, the baserate, or the prior probability, which is the probability of finding a librarian or a science major in the community, should be considered. In other words, one needs to know the number of librarians and the science majors. In a medical environment, in order to answer the common question "what is the probability of a particular outcome?" the clinician needs to recall other patients with similar characteristics and know the base rate. A key advantage of a CBR-based DSS is in providing information about similar cases. However, in order to avoid a judgment error the physician will still need to know and consider the base rates for a particular outcome in the population the patient comes from. This prior probability is likely judged by the physician but caution should be exercised given the tendency of decision makers not to consider the base rates.

\subsubsection{Confirmation Bias}

Another bias stemming from the representativeness heuristic is overconfidence when dealing with redundant predictors. An example of such a situation leading to error is given by Sox (1988) where the predictors of a disease always occur together. Under such circumstances, the clinician is often unjustifiably confident in his or her judgement. Given that several characteristics of a disease could occur together, the presence of one should be enough to know that a disease exists, and the presence of additional characteristics should not increase confidence. In this case, the presence of additional characteristics enhancing their overall representativeness increases the estimate of the probability that an outcome has occurred and may even lead to disregard for other salient information. Typically, the more information available to a decision maker, the more confident she becomes in her judgement. However, Oskamp (1965) reported a study in which increasing amounts of information led to increased 
confidence in judgement but did not improve accuracy. Hence, non-independent bits of information seemed to enhance confidence without enhancing accuracy (Oskamp, 1965).

As well, confirming evidence is often seen as more pertinent than non-confirming evidence. This so-called confirmation bias, also termed the illusion of validity, represents a preference for information that is consistent with one's hypothesis over information that is inconsistent with it (Mynatt, Doherty, \& Tweney, 1977). Confirmation bias is quite widespread and reportedly difficult to eliminate. The use of similarity-matched cases by a DSS to help with outcome prognosis might serve to either introduce or support the confirmation bias of clinicians who have formed an initial hypothesis about the outcome of a patient and then seek further confirmation by reviewing the outcomes of 10 other patients with similar characteristics. This aspect of judgment will be examined with the neonatologists in this thesis through interviews.

\subsubsection{DSS and Medical Decision Making}

Decision makers assume that the probability of events that have already occurred provides a good estimate for the probability of future events, under similar circumstances. The underlying assumption is that future probabilities are predictable from past probabilities. Although not perfect, this is a robust assumption and much research has shown that it can produce almost perfect predictions (McFall \& Treat, 1999). Hence, the DSS can help decision makers by providing them with a historical probability of outcome under similar circumstances.

This section has reviewed errors in judgment that occur due to use of various heuristics, or shortcuts, in decision making. The potential effects on decision making of those 
employing a CBR-based DSS have been discussed. The confirmation bias, one of the likely sources of error with the use of this system will be examined by this thesis.

\section{RESEARCH ON DSSS}

Much information is available about the technical and theoretical aspects of the CBR in clinical decision making and there is some evidence suggesting that DSS's in general can improve clinician performance (Klaus-Dieter et al., 1998; Seitz, Uhrmacher, \& Damm, 1999; Lisboa, 2002). Lisboa (2002) has reviewed the evidence for health benefits in medical interventions arising from the use of ANNs in DSSs in several medical domains. The data collection and findings were based on Randomized Controlled Trials and Clinical Trials

publications in the PUBMED database (www.ncbi.nlm.nih.gov/PubMed). Reviews of computer-based clinical DSSs conclude that overall the systems with most potential are those used for preventative care, such as DSSs intended to assist with drug dosage and warning against adverse effects from prescription drugs. Other DSSs have also proved useful. Lisboa points out that he has found no reports with evidence of benefits from inference-based DSS involving severity of illness scores despite their wide use, probably because of the difficulties involved in conducting such studies in clinical settings in which a decision system can actually be used by practitioners.

There are three general categories of DSSs involving NNs in medicine.

1- Tools for attention focusing.

2- Tools used for patient assessment, prognosis, and advice. 
3- Interactive tools for scenario analysis and hypothesis generation.

The first general category involves DSSs used mainly for pattern recognition and detection of abnormalities from hospital based information or laboratory systems. For example, computerassisted evaluation of mammograms has reduced the number of false negative results by $50 \%$, although the DSS use increased the required time for making a diagnosis and added to the medical staffs training time and the required equipment (Lisboa, 2002). The use of DSS in cervical cytology for review of Pap smear slides was the first patented medical application of an NN, called PAPNET, and it resulted in significant improvement in accuracy in detection of severe cytological abnormalities (Boon \& Kok, 1995). The drawbacks and issues involved in the application of these systems involve the quality of the underlying data, especially missing values in databases, which is a frequent occurrence.

The second general category of NN based DSSs involves tools used for patientspecific assessment, prognosis, and advice. These often involve access to electronic databases and recall of historical patient records that are the most relevant for a specific patient query. The CBR falls in this category as are DSSs involving survival analysis. Survival analysis is a statistical method using hazard rates, also called force of morbidity, which defines a patient's probability of death, or other identified outcome, within a particular time interval (Szklo \& Nieto, 2000). The use of this category of DSS is most common in the field of oncology. For example, they are frequently used to help clinicians with mortality prognosis and prediction of post-surgery recurrence of disease for patients with breast and colorectal cancers. However, their use has not been demonstrated to significantly improve the predictive power of more traditional statistical analyses such as those using the Baye's Theorem. It has been argued that 
their advantage is in their complementary use alongside classical survival analysis (Lisboa, 2002).

The final and third category involves interactive tools for scenario analysis intended to allow clinicians to gain new insights and generate better hypotheses. In a study designed to examine the effect of computer-based consultation on clinicians' diagnostic reasoning, it was found that DSSs can positively influence the diagnostic reasoning of clinicians and that medical students are the most likely user group to benefit from such systems (Friedman et al., 1999). The study focused on how DSSs can improve clinicians' ability to generate correct diagnostic hypotheses and not on the correctness of diagnostic suggestions offered by the DSS. Although Friedman's study concerns diagnostic reasoning and not prognosis, it does illustrate the use of DSSs. Although the computer generated the correct diagnosis only $41 \%$ of the time, the clinicians were still able to benefit from using the system and improve their diagnostic abilities. Friedman et al. suggest that a DSS does not have to be correct at all times to be helpful as long as the computer system's advice is filtered through human cognition. In their experiment, medical students generated a correct diagnosis on their own $26 \%$ of the time. After consulting the decision aid, correct diagnoses were changed into wrong ones $6 \%$ of the time, but wrong diagnoses were changed into correct ones $15 \%$ of the time. Experienced clinicians made a correct diagnosis on their own $49 \%$ of the time. After consulting the decision aid system, they changed a correct diagnosis into a wrong one $7 \%$ of the time and an incorrect diagnosis into correct one $10 \%$ of the time. The results for resident clinicians fell somewhere between the results of the other two groups as shown in Table 2 . These results suggest that while medical students benefited most from using the decision aids, experienced clinicians who were more likely to generate correct diagnosis without the help of decision support were also 
more likely to change their once correct diagnosis into wrong ones after using the decision aid. In other words, experienced clinicians were more likely to be fooled by the system. Although the data in Table 2 were given by Friedman and his colleagues they were not specifically discussed. Their analysis did show a modest significant improvement in overall diagnostic reasoning of clinicians, that is for students, residents, and experts combined. This study demonstrates that although a DSS can be useful, caution should be exercised with its use in terms of its intended audience and purpose.

Table 2. Effects of Using Decision Support on Correct Diagnosis in \%

\begin{tabular}{ccccc}
\hline $\begin{array}{c}\text { Decision } \\
\text { Maker }\end{array}$ & $\begin{array}{c}\text { \# correct } \\
\text { diagnoses before } \\
\text { use }\end{array}$ & $\begin{array}{c}\text { \# correct } \\
\text { diagnoses after } \\
\text { use }\end{array}$ & $\begin{array}{c}\text { Improvement in \# } \\
\text { of correct } \\
\text { diagnoses }\end{array}$ & $\begin{array}{c}\text { Decline in \# of } \\
\text { correct diagnoses }\end{array}$ \\
\hline Students & 25.8 & 34.9 & 14.8 & 5.7 \\
Residents & 43.7 & 49.1 & 10.8 & 5.4 \\
Faculty & 49.1 & 52.4 & 10.3 & 7.0 \\
\hline
\end{tabular}

The MIRG, among others, have suggested that a CBR-based DSS can be helpful as a prognostic tool in a clinical setting. They suggest that the CBR can be employed as a memory aid, and that the similarity-matched cases it provides in response to a query can improve clinicians' ability to prognosticate. The improvement is instigated by increasing the available amount of information, or memory, and it is argued that remembering more cases and having more information can help decision making (Frize \& Walker, 2000). Others have suggested that the use of a $\mathrm{CBR}$ is particularly relevant to medical decision making because expert knowledge consists of a combination of rules and experience (Schmidt et al., 2001). It is not 
clear however, how historical information about the condition and outcome of other patients might act as experience for the clinicians viewing this information. $A$ review of the $C B R$ literature did not reveal why or how knowledge of another clinician's experience might act as actual experience for those who come to know about the case given that human decision making entails many considerations of which memory is only a part.

\section{PHYSICIANS' USE OF DECISION SUPPORT SYSTEMS}

This section will consider potential barriers to adoption of DSSs by physicians and looks as some of the ideas already explored within the relevant literature. It will suggest ways of understanding these barriers which could potentially help in making DSSs more effective.

Despite the advances in medical technology and improved medical knowledge the practice of medicine remains vulnerable to human error. Many hoped that computer-based technology can, if not render decision makers infallible, compensate for some of the decision flaws and consequently decrease health care costs. Many medical associations and government agencies as well as various well-intentioned groups recommend the use of an assortment of computer-based systems for clinical performance enhancement and decision error reduction but, such recommendations do not guarantee implementation or adoption by the clinicians.

In general, many physicians view technology to be too expensive or unusable by the health care systems that are in place (Schoen, Osborn, \& Blendon, 2000). Electronic medical records, other than those used for administrative purposes, are slowly being adopted by hospitals and primary care practitioners mostly because they are perceived to cut costs and to make patient records more accessible. Ease of access and ability to modify patient records 
electronically is believed to improve patient care and reduce medical errors because doctors will be able to have more accurate records and better access to those records. Potential barriers to a quick adoption of electronic medical records have been identified as problems with data entry methods and confidentiality concerns about patient data. For physicians to adopt such systems, they need to trust and perceive them as useful tools that will improve the quality of health care (Wager, Lee, White, \& Ward, 2000).

Many DSSs are available to the medical community for a variety of purposes, but a lack of trust and acceptance of technology has been reported to be the main reason for the limited use of some of these systems (Smith \& Geddes, 2003). A lack of trust limits system use resulting in underutilization or complete rejection even of those that are installed and available. Smith and Geddes (2003) suggest this lack of acceptance may be due to clinicians' resistance to change and their fear that perhaps the technology might replace the experts and affect their livelihood. Others have suggested similar reasons (Sheridan \& Thompson, 1994). The lack of trust and acceptance may also be due to a weakness in the system, real or imagined, and doubts about its expected performance or the way the system could influence clinical work. Either way, DSSs can be of value only if they can be trusted, accepted, and employed.

There may be ways to bring about this trust and acceptance. Chiefly, a user-centered approach to DSS development may prove useful. If clinicians were involved in the planning and development stages of software development they may be more accepting of it in their practices because their needs can potentially be incorporated. In addition, an appreciation of the clinicians' needs and opinions will improve the development process of any DSS and physicians will be more likely to trust it. In order to examine the clinicians' views and attitudes 
toward the use of a CBR based DSS and their underlying data, this thesis explores the opinions and attitudes of neonatologists about DSSs, medical databases, and predictive models through interviews.

\section{MEDICAL DATABASES}

As stated earlier, DSSs rely on medical data to operate. Medical databases such as the NICU database are developed mainly for the purpose of assessment of the efficiency of health care in hospitals. They are also used for extraction of various data types for purposes other than assessment of effectiveness. The hospital staff responsible for data entry is required to follow guidelines which provide definitions of variables and give instructions on how each variable should be measured, calculated, or entered. Data entry is not always executed flawlessly. There is evidence suggesting some of the inaccuracies in the data contained in medical databases are due to simple errors such as typos, and others are due to errors of judgement. For example, a study of the Dutch National Intensive Care Evaluation Registry examined the data of the registry for errors (Arts, Bosman, Jonge, \& Keizer, 2003). They reported three main types of errors in the registry. These were incompleteness of information (7.4\%), non-adherence to data definitions such as ignoring guidelines $(11.4 \%)$, and errors that could not be accounted for, involving mostly cases where documented data did not correspond to true data (2.1\%). Data items with accuracy levels below $75 \%$ were measurements of body temperature (18\% accurate), blood gas calculations ( $23 \%$ accuratc), mean arterial blood pressure ( $42 \%$ accurate), respiratory rate $(52 \%$ accurate), and urine output (70\% accurate), among others. These particular items are reported here especially because they are part of the predictive models used by the DSS considered for this study. Arts and 
colleagues (2003) report that training of physicians and hospital staff on data extraction for input into the registry resulted in improvement in mortality prediction from severity of illness scores.

In a separate study at the University of Vermont College of Medicine, a database for infants with very low birth weight was assessed for data accuracy (Horbar \& Leahy, 1995). The database contained medical records of over 4000 low-weight infants. A random sample was selected from the database and checked for accuracy. Accuracy was determined by agreements between medical records and the database. The analysis revealed a number of discrepancies, $9.3 \%$ of which were data entry errors and the rest, $90.7 \%$, were due to interpretation and transcription errors. In this study, researchers were able to improve the quality of the data after introducing quality control procedures including visual inspections and improved data entry measures by those responsible for completing data forms. Horbar and Leahy (1995) concluded the database is reliable.

Studies about data accuracy in the Canadian NICU are not currently available and there is no information on whether those responsible for collection of information and data entry in the NICU database receive specific training. The version of the NICU database used by the CBR has information about 20,488 patients. Many of the variables have missing values for more than the 200 information categories that make up a patient's record. The majority of the data types are numerical, some are categorical, and there are time, date, and string (character) data types. Not all information categories have been filled in because not all babies require all tests. In general, the sicker the patient, the more information there is, presumably because more physiological measures are gathered for sicker patients. 
A preliminary examination of a random sample of 1000 cases from the NICU database showed that $75 \%$ of the values for the variables used in the SNAPPE-II score were missing. These missing variables are not necessarily due to errors. They were probably not recorded because they were not needed and therefore not collected. A visual inspection of the data revealed a number of typos such as missing commas or dots, for example turning a value of 7.60 to 760 . The MIRG at Carleton University has been working with this database extensively and has corrected many of these errors, therefore producing a cleaner database. However, clinicians' perception of trustworthiness of a Canadian database of this size is perhaps as important as the actual accuracy of the data it contains. This thesis examines such perceptions through interviews with neonatologists.

\subsection{Skewed Distribution of Cases}

The prevalence of death in the NICU database used in this thesis is low, causing a skewed distribution of cases. Furthermore, the NICU database contains more information for sicker patients because they require more tests and more therapy than the less sick patients. The database used to develop the MIRG model, and used here for testing of predictive ability of the models, contains only cases without missing information. It is likely that the cases without missing values belong to the sicker patients, and it is plausible that the sicker patients are more likely to die. This is an important issue to consider given that the ANN's are trained with complete (no missing values) patient cases. Hence, it is possible that the ANNs are trained to overestimate mortality. In order to examine this aspect of the database and the DSS low birth weight infants, that is all cases that are presumably at a greater risk of death, will be selected as a sub-database and analyzed separately to re-examine the predictive ability of the models with a less skewed population distribution. 


\subsection{Predictive models}

In order for the DSS and a medical database to cooperate, the system needs to be given a set of variables based on which the CBR and the NN respond to a query. Recall that in order to calculate a SNAPPE-II score a dataset is extracted from each patient record, and the severity of illness scoring system (SNAPPE-II) is used to calculate a score for each patient based on the values in the dataset. This score quantifies the severity of illness and the probability of in-hospital mortality of that patient. Recall also that the final score arrived at by this index is not used by the predictive models discussed here, but the variables are. The reasons for choosing the variables from this particular severity-of-illness measure for use by the DSS were given by Ennett (2003). The reasons were that SNAP-II (the precursor to SNAPPE-II) has proven to be effective in predicting morbidity and has resulted in areas under the Receiver Operating Characteristic (ROC) curve of .84 to .92 .

The ROC methodology is a statistical procedure derived from electronic signal detection theory (Hanley, 1989). Its use is becoming more frequent in the field of psychology (Swets et al., 2000) and has often been applied to medical applications. This method is used to evaluate the accuracy of predictions against actual outcomes by considering sensitivity, that is the rate of true positives, and specificity, the rate of true negatives. When the standardized true positive rates (i.e., hits) are plotted against the false positive rates (i.e., false alarms or 1specificity) an ROC curve is obtained. The area under the ROC curve is interpreted as the measure of diagnostic accuracy, or more specifically, the probability of correctly choosing the positive case from a pair of cases each randomly chosen from the sets of positive and negative 
cases, respectively. The area under the ROC curve for SNAP-II reported by Ennett (2003) is considered high.

Ennett has developed a new neonatal mortality model, called the MIRG model, that is based on the original SNAP severity-of-illness measure. Other neonatal mortality models are available in the medical literature, with areas under the ROC curve even higher than that given by the SNAP-II model. These models, however, can only be used with more restrictive populations such as infants of low birth weight, extremely low birth weight, or preterm neonates (Ennett, 2003). Ennett proposed a model that could be used with neonates of all gestational ages and birth weights. Using a neonatal dataset with missing values imputed by the ANN-CBR hybrid, ANN weight-elimination methods were employed to identify a new set of variables that can predict neonatal mortality. Full details of ANN methods used to arrive at the final model can be found in Ennett's $\mathrm{PhD}$ thesis (2003). In summary, the model started with 30 of the 37 variables in the SNAP model. Variables with lowest weights were eliminated (or "pruned") and the classification performance, that is the ability to correctly predict outcome, of the remaining variables was examined. The process was repeated until further variable elimination failed to improve the classification performance. In this way, 13 variables were selected as part of the new neonatal mortality model. Ennett calls this new set of variables, the "MIRG model", and introduced it as a new neonatal mortality model that can outperform the SNAPPE-II severity of illness model in terms of its ability to predict patient outcome more accurately.

The ANN method used by Ennett is similar to statistical stepwise and backward elimination methods used in regression analyses. It is worth noting that stepwise and backward 
elimination methods used by various regression techniques for predictor-selection are often unreliable and can capitalize on chance (Menard, 2002; Pedhazur, 1997; Zhang \& Singer, 1999). The appropriate and frequently used methods of developing and testing the predictive

models are ANN's and logistic regression (Terrin, Schmid, Griffith, D'Agostino, \& Selker, 2003), though others have suggested similar but newer statistical techniques (Zhang et al., 1999). This thesis will use the statistical method of logistic regression to test and compare the predictive ability of the SNAPPE-II and the MIRG models. It will also examine perceptions and views of neonatologists regarding the variables that they consider as being predictive of infant mortality including those used by these models.

\section{SUMMARY OF INTENT AND QUESTIONS}

The MIRG's DSS is not currently in use in a clinical setting. In order to introduce this DSS in a clinical setting it is important to know about the physicians' requirements, beliefs, and attitudes toward DSSs and about some of the potential heuristic biases that could arise from the use of a DSS. It is also important to know which variables would be chosen by the experts themselves for prognostic decision making. This knowledge would facilitate the introduction of the system in clinical settings, identify potential barriers to its use, and possibly overcome its shortfalls from the physicians' perspective.

The MIRG's DSS relies on a NICU database. Physicians' opinions of this database, their beliefs about the accuracy of its data, and the usefulness such database for predicting patient outcome will play a role in their trust and acceptance of the DSS. 
In addition, recall that the 9 SNAPPE-II variables were chosen as the predictive model for this DSS, and also that Ennett (2003) has subsequently proposed another predictive model, the "MIRG model", consisting of 13 variables, some of which overlap with the SNAPPE-II model. In order to fully understand the merit of the variables used by these two predictive models, their actual prognosticity will be examined. Their actual prognosticity can then be compared with the physicians' perception of their prognosticity.

Two types of analyses will be undertaken for this thesis. One involves an exploratory, qualitative investigation of physicians' perception of prognostic DSSs, their potential acceptance, and their predicted use of the system. The other involves a set of quantitative statistical analyses that examine the predictive ability of the SNAPPE-II and the MIRG models with respect to actual neonatal mortality outcomes.

\section{METHOD}

The method is outlined in two sections below. The first describes a series of semiformal interviews conducted with neonatologists in Ottawa. Each interview had two main objectives. First, it sought information about the perceptions and attitudes of neonatologists toward prognostic DSSs for NICU's. Second, it sought their opinion about the prognosticity of the variables used by the two models, the SNAPPE-II and the MIRG models, which have been suggested by the MIRG as the potential predictive models to be used by a prognostic DSS.

The second section describes multiple logistic regression analyses of the 9 SNAPPE-II and the 13 MIRG variables. These were carried out to examine the variables individually and 
within each of the models. The variables and the two models were evaluated for their prognostic ability. As well, all pairs of variables were examined to determine the presence of any two-way interaction effects. This section also compares the two models in order to identify the one likely to perform best in terms of the accuracy of neonatal mortality predictions.

\subsection{Interviews}

In order to examine the perceptions and attitudes of neonatologists toward computer use and prognostic DSSs in general, 7 neonatologists were interviewed. Participants were asked about their familiarity with the concept of prognostic DSSs and were provided with a verbal explanation (read by the interviewer) about a hypothetical prognostic DSS in order to make sure there was a shared understanding among both the participants and the interviewer about the CBR-based prognostic DSS and the nature of the output that it would provide. At this point, no information was provided about the type of variables or the kind of information that would be used by such a DSS, only that it would search for sets of cases matched for similarity to an inputted case and would present mortality outcomes for each of those cases. Subsequently, participants were asked questions concerning their attitudes toward a prognostic DSS, and their opinions concerning the prognosticity of the variables used by the SNAPPE-II and the MIRG models.

The first set of questions was about participants' general views and beliefs about the value of a prognostic DSS in the NICU, and their willingness to interact with and use a DSS. The next set examined participants' level of acceptance of a prognostic DSS' output in terms of its accuracy, reliability, and trustworthiness. These questions intended to identify participants' views about the information gained from a prognostic DSS' output, as well as 
their views about the fact that the information source is a medical database obtained from hospital NICU's. The next set of questions dealt with the issue of confidence. Participants were asked if the information gained from a prognostic DSS' output would affect their confidence in their own hypothesis about the mortality outcome of a neonatal patient, both when the output agreed and when the output disagreed with that hypothesis. This question was intended to examine the manner in which participants might view confirming versus disconfirming information when it is provided as output from a prognostic DSS.

The last set of questions dealt with the variables. Participants were asked their opinion concerning what they believed to be the three most important prognostic variables for neonatal outcomes, the kind of information that they would "like" a prognostic DSS to provide, and what sort of information they perceived to be predictive of mortality. They were then asked to explicitly consider the variables from the two predictive models, to rank order them, and to add any other variables they deemed to be important.

\subsection{Procedure}

Seven of the 9 Ottawa neonatologists from the Ottawa Hospital campuses and the Children's Hospital of Eastern Ontario (CHEO) were interviewed. One of the neonatologists is associated with the MIRG and the development of the prognostic DSS and was not interviewed. Another was not available to take part. After obtaining participants' written consent, a tape recorder was used to record the interviews. Nine of the interview questions were open ended, others close ended in the form of check boxes and ranking questions (see Appendix A for interview protocols). Participants were first asked to fill a short form about the length of their experience in neonatology, and their self-rating of expertise in computer 
use. They were also asked about the types of computer applications they use and the purposes for which they use a computer. These questions were asked to compare the level of experience of respondents in the field of neonatology and their computer skills to their perception of technology. Next, participants were asked to answer nine open-ended questions and then rank 15 variables in terms of their importance. Before the end of an interview session, participants were asked for any other information they thought might be useful to the study. Further suggestions or comments were noted.

All participants received a debriefing sheet at the end of the interview. The interviews were tape recorded and transcribed ad verbatim. Each interview session was conducted with one neonatologist only and lasted approximately 40 minutes.

\subsection{Model Evaluation}

This section gives a brief overview of the use of logistic regression for model evaluation. It should be emphasized that the aim of these analyses is to evaluate two different models, not to propose a new predictive model. The procedure followed is one of fitting and testing, not model building.

Two sets of source data, the MIRG and SNAPPE-II datasets, were obtained from the MIRG of Carleton University. These datasets originated from the database of the NICU Network and although they represent only a portion of the entire database they were comprised of rather large sample sizes of 5102 and 2437 cases, respectively. The data in these two datasets were examined and any obvious outliers were removed. The two datasets were then linked (merged) to create a third dataset, the composite dataset, which contained a full set of variables from the two models combined. The three datasets were compared and 
descriptive information for all sets was noted. All logistic regression analyses were performed using these datasets.

In general, regression methods are used to describe the relationship between dependent (outcome) and independent (predictor) variables. When the dependent variable is dichotomous (takes two discrete values), logistic regression methods are used to describe the relationship between the two possible outcomes and the independent predictors. The goal of logistic regression is to find the best fitting and the most parsimonious model that describes this relationship. In logistic regression, the distribution of the outcome variable follows a binomial distribution. This distribution involves the conditional probabilities of observing one of the two possible outcomes, given the observed values of the independent variables. The key quantity in logistic regression is the log likelihood obtained from the likelihood function. The likelihood function states the likelihood of the full set of all observed outcomes as a function of a number of unknown parameters that, in this case, are regression coefficients (betas). Maximizing this likelihood function involves an iterative process that estimates the parameter values which have the closest fit with, or are most likely to give rise to, the observed outcomes (Hosmer \& Lemshow, 2000).

The likelihood for any observed case is the probability of observing either an occurrence $(\mathrm{Y}=1)$ or a non-occurrence $(\mathrm{Y}=0)$ where in the following analyses occurrence will refer to living (or, similarly, to not dying). In order to avoid the need to multiply these probabilities when obtaining an overall likelihood for all of the cases together, the likelihood function is transformed into a $\log$ likelihood function. The $\log$ likelihood function varies from negative infinity to zero, where the closer the likelihood is to 1 , the closer the log likelihood 
value is to 0 . Hence, a perfect model would have a log likelihood of 0 . In order to make the interpretation of the log likelihood easier, the values are multiplied by -2 . This transformation changes the range of the log likelihood from 0 to positive infinity. It also gives the likelihood function a distribution similar to the chi-square distribution (Menard, 2002).

Evaluation of logistic regression models requires a comparison of likelihoods. Typically, the log likelihood for the full model is compared to a log likelihood value associated with a baseline model that comes from assuming that all beta coefficient parameters are equal to 0 (a model which contains only the intercept, or constant, term). When these two log likelihoods are multiplied by -2 , the difference between them is approximately distributed as a chi-square distribution, with a degrees of freedom equal to the number of variables, which can also be used to test the null hypothesis $\mathrm{H}_{0}: \mathrm{B} 1=\mathrm{B} 2=\ldots=\mathrm{Bk}=0$ (Pampel, 2000). Note that this general procedure can be used to compare the fits of two models containing any number of variables as long as one of the models is completely nested within the other.

SPSS Version 10.0 was used to perform the logistic regressions. The beta coefficients were estimated through the reiterative process of maximizing the likelihood function. The relation of the independent variables to the outcome variable was examined in two ways. First, both the fits of the overall models and the contributions of the individual variables to the models were evaluated statistically. Next, the observed outcomes were compared with the predicted outcomes. In order to compare the predictive abilities of the two models the ROC method was used. The predictive ability of a model lies in its potential to correctly differentiate between two outcome alternatives and to select the correct outcome. With a predicted binomial dependent variable and two possible states of the world, there are four possible 
outcomes. The first two are the selection of the correct outcomes, the true positives and the true negatives. The other two are the selection of the incorrect outcomes, the false positives and the false negatives. A model's ability to correctly predict the rarer outcome (hit) is termed the sensitivity of the model. A model's ability to correctly predict the more common outcome (correct rejection) is termed the specificity of the model. AN ROC analysis compares sensitivity against 1 - specificity.

\section{RESULTS}

\subsection{The Datasets}

Two datasets were used. The MIRG dataset consisted of 2,437 cases with values for each of the 13 variables of the MIRG model. The SNAPPE-II dataset had 5,102 cases with values for each of the 9 SNAPPE-II variables. Both datasets included two additional columns, the outcome (life/death) column and a "case-link" column as an internal ID. In each dataset, all cases had values for all variables, there were no missing values. For comparison purposes, a "composite" dataset that consisted of 2429 cases with values for all 15 of the SNAPPE-II and the MIRG variables combined was derived from these two datasets.

The outliers in the SNAPPE-II data source were identified based on Mahalanobis distance, where the majority of cases fell below a Mahalanobis distance of 75 . Seventeen cases with values of 75.02 and above were filtered from the SNAPPE-II dataset. The outliers in the MIRG dataset were also identified based on Mahalanobis distance, where the majority of cases also fell below a Mahalanobis distance of 75 . Twenty four cases with values above 75.28 were filtered from the MIRG dataset. The outliers in the composite dataset were identified based on Mahalanobis distance as well, where the majority of cases fell below a Mahalanobis distance of 
75. Twenty seven cases with values above 75.28 were filtered from the composite dataset. After removing the outliers, the non-mortality rate was changed from 89.5 to $89.7 \%$ for the MIRG dataset but remained unchanged for the SNAPPE-II dataset at $90.5 \%$. Summary information about the Mahalanobis distance is presented in Table 3. Table 4 provides a descriptive summary of databases used in the analyses after the outliers were removed.

Table 3. The Mahalanobis Distance for Datasets

\begin{tabular}{lccccc}
\hline Model & N & Min & Max & Mean & Std. dev. \\
\hline SNAPPE-II & 5102 & .312 & 524.763 & 8.998 & 13.222 \\
MIRG & 2437 & 1.506 & 621.279 & 12.994 & 17.847 \\
Composite & 2429 & 1.814 & 627.151 & 14.993 & 19.109 \\
\hline
\end{tabular}

Table 4. Database Summary Information with Outliers Removed

\begin{tabular}{lccc}
\hline Database & Number of Variables & Number of Cases & $\%$ Non-mortality \\
\hline SNAPPE-II & 9 & 5,085 & 90.5 \\
MIRG & 13 & 2413 & 89.7 \\
Composite & 15 & 2402 & 89.5 \\
\hline
\end{tabular}

10.2 Testing the Significance of the Models

Two approaches were taken to test the fit and adequacy of the models. First, the full models were each tested against a baseline intercept-only model. This test determines whether the independent variables in each of the models as a whole are significantly related to the 
outcome variable. Second, the significance of the contribution of each variable to their models was tested individually.

10.2.1 Approach 1: Assessing Significance of Variables in the Models as a Whole

In logistic regression, the maximum likelihood method yields estimates for the unknown parameters, through a reiterative process. In other words the outcomes are known, but the variable beta coefficient parameter values that give rise to the observed outcomes are unknown. The maximum likelihood method starts with an initial estimate for each coefficient, and then adjusts them in order to obtain the smallest $-2 \log$ likelihood value for the model.

The model testing approach that is then used is to compare the model with all of the variables included (full model) to a model containing only the intercept term (base model). This method of testing for the overall significance of the variables in the model is called a test of the goodness of fit of the model. These tests make use of the difference between the values of the $-2 \log$ likelihoods for the full and base model, which are analogous to the sums of squares error and the sums of squares total in linear regression, respectively. This difference value can be interpreted as a chi-square statistic and, hence, can be used to test of the null hypothesis that none of the variables are significantly related to the outcomes.

To examine the goodness of fit for each of the SNAPPE-II, MIRG, and composite models, the chi-square statistics for the "Omnibus Tests of Model Coefficients" were obtained for each data set. These chi-squares were significant at the .001 level for all three models, indicating that, for each model, the addition of all of the independent variables as predictors to the base model helped in predicting the outcome probabilities. Table 5 gives the summary 
statistics from these tests. Table 6 gives the results that were obtained when these same tests were performed using only cases from the composite dataset.

Table 5. Omnibus Tests of Model Coefficients in the Different Datasets

\begin{tabular}{lccc}
\hline Dataset & Model Chi-square & df & Sig. \\
\hline SNAPPE-II & 803.491 & 9 & .001 \\
MIRG & 395.801 & 13 & .001 \\
Composite & 398.119 & 15 & .001 \\
\hline
\end{tabular}

Table 6. Omnibus Tests of Model Coefficients for Different Models Using the Composite Dataset.

\begin{tabular}{lccc}
\hline Model & $\begin{array}{c}\text { Model Chi- } \\
\text { square }\end{array}$ & df & Sig. \\
\hline SNAPPE-II & 303.915 & 9 & .001 \\
MIRG & 392.134 & 13 & .001 \\
Composite & 398.119 & 15 & .001 \\
\hline
\end{tabular}

In logistic regression, an analogue to the linear $R^{2}$ is the Likelihood Ratio $R^{2}\left(R_{L}^{2}\right) \cdot R_{L}^{2}$ indexes the proportional reduction in the value of the $-2 \log$ likelihood for the full model in comparison to the base model (Menard, 2000) and, like $\mathrm{R}^{2}$, ranges between 0 and 1 . The value for the $\mathrm{R}_{\mathrm{L}}^{2}$ is not reported by SPSS and was therefore calculated by hand and reported in Table 7 , along with an alternative quantity that is reported by SPSS, the Nagelkerke $\mathrm{R}^{2}$ (Menard, 2000) for the $-2 \log$ likelihood results obtained for each model in its own dataset. 
Table 7. Measures of Association between the Predictors and the Outcome for the Different Datasets.

\begin{tabular}{lccccc}
\hline Model & Initial-2 LL & $\begin{array}{c}\text { Model-2 } \\
\mathrm{LL}\end{array}$ & $\begin{array}{c}\text { Chi-Square } \\
\text { difference }\end{array}$ & $\mathrm{R}_{\mathrm{L}}^{2}$ & $\begin{array}{c}\text { Nagelkerke } \\
\mathrm{R}^{2}\end{array}$ \\
\hline SNAPPEII & 3183.567 & 2380.076 & 803.491 & .252 & .314 \\
MIRG & 1637.826 & 1222.137 & 395.801 & .253 & .312 \\
Composite & 1596.568 & 1209.843 & 391.119 & .253 & .318 \\
\hline
\end{tabular}

\subsubsection{Model Comparison}

Given that the SNAPPE-II model is not nested within the MIRG model (the SNAPPE-II model is not a subset of the MIRG model) direct comparisons of the goodness of fit of the two models are not possible. It is however possible to compare the fits of both the SNAPPE-II and the MIRG models to the fit of the composite model because they are both nested within the composite model. This comparison involves testing whether the addition of the extra variables in the composite model to each of the SNAPPE-II and MIRG models results in a significant reduction in the $-2 \log$ likelihood (by comparing this difference to a chisquare distribution) and is conceptually equivalent to an $\mathrm{R}^{2}$ change test in linear regression. The results of these tests showed that the addition of the extra 6 composite variables to the SNAPPE-II model significantly increased the model fit (Table 8) and also that the addition of the extra 2 composite variables to the MIRG model significantly increased the model fit (Table 9). 
Table 8. Comparison of the Model Chi-squares for the SNAPPE-II model and the Composite Model.

\begin{tabular}{lccc}
\hline Model & Chi-square & df & Sig. \\
\hline SNAPPE-II & 303.95 & 9 & .001 \\
Composite & 398.119 & 15 & .001 \\
Change & 94.205 & 6 & .001 \\
\hline
\end{tabular}

Table 9. Comparison of the Model Chi-squares for the MIRG model and the Composite Model.

\begin{tabular}{lccc}
\hline Model & Chi-square & $\mathrm{df}$ & Sig. \\
\hline MIRG & 392.134 & 13 & .001 \\
Composite & 398.119 & 15 & .001 \\
Change & 5.985 & 2 & .050 \\
\hline
\end{tabular}

\subsubsection{Comparing the Models with Low Birth Weight Cases Only}

The composite dataset contains 503 cases with birth weight values of less than $1000 \mathrm{~g}$. Hence, these cases were isolated and used to compare each of the two predictive models with the composite model. Results indicated that the addition of the extra composite variables to both the SNAPPE-II model (Table 10) and the MIRG model (Table 11) resulted in a significant increases in model fit for these cases. 
Table 10. Comparing the SNAPPE-II and the Composite Models for Low Birth Weight Data Only.

\begin{tabular}{lccc}
\hline Model & Chi-square & df & Sig.. \\
\hline SNAPPE-II & 101.927 & 9 & .001 \\
Composite & 131.778 & 15 & .001 \\
Change & 29.852 & 6 & .001 \\
\hline
\end{tabular}

Table 11. Comparing the MIRG and the Composite Models for Low Birth Weight Data Only.

\begin{tabular}{llll}
\hline Model & Chi-square & df & Sig. \\
\hline MIRG & 118.682 & 13 & .001 \\
Composite & 131.778 & 15 & .001 \\
Change & 13.096 & 2 & .001 \\
\hline
\end{tabular}

10.2.2 Approach 2: Assessing the Significance of the Variables in the Model Individually

In logistic regression, the non-linear relationship between the independent variables and the dichotomous dependent variable is linearized. Therefore, the reported coefficients cannot be interpreted in the exact same way as linear regression coefficients (Pampel, 2000) because logistic regression coefficients actually represent logged odds. Taking an antilog of a coefficient $(\exp (B))$ converts the coefficient value into an odds ratio value. This odds ratio value then indicates the change in the odds of observing the outcome $\mathrm{Y}=1$ (living) for each unit change in the predictor variable.

The approach used to test the significance of the coefficients for each of the predictor variables involves the Wald test (Menard, 2000). The Wald test is obtained by comparing the 
estimated maximum likelihood coefficients to their estimated standard error. The result is a ratio with a standard normal distribution. It tests the null hypothesis $B_{k}=0$ (i.e., $\exp \left[B_{k}\right]=1$ ). The advantage of this approach is the Wald test's ability to provide a significance test for each of the coefficients in the model. Table 12 shows the results of these tests for all three models with the composite dataset as the data source because it allows for direct comparisons of the results from each of the models. The results showed that not all of the tests for the estimated regression coefficients were significant, using either a standard alpha or Bonferroni-corrected alpha, and also that whether a test of a particular regression coefficient was significant sometimes depended on which model was being used. Most notably, two key SNAPPE-II variables, $\mathrm{SGA}$ and $\mathrm{PO}_{2} \mathrm{FIO}_{2}$, did not contribute significantly to the model once the six extra MIRG variables were added. However, both the LBloodP and HBloodP variables were nonsignificant predictors in either their own models or in the composite model. 
Table 12. Individual Tests of the Significance of the Variables in Models using the Composite Dataset

\begin{tabular}{|c|c|c|c|c|c|c|c|}
\hline \multirow[t]{2}{*}{ Variables } & \multirow[t]{2}{*}{$\mathrm{df}$} & \multicolumn{2}{|c|}{$\begin{array}{c}\text { SNAPPE-II } \\
\text { model ( } 9 \\
\text { variables) }\end{array}$} & \multicolumn{2}{|c|}{$\begin{array}{l}\text { MIRG model (13 } \\
\text { variables) }\end{array}$} & \multicolumn{2}{|c|}{$\begin{array}{l}\text { Composite model } \\
\text { (15 variables) }\end{array}$} \\
\hline & & Wald & Sig. & Wald & Sig. & Wald & Sig. \\
\hline LSERUM & 1 & 21.68 & .001 & 18.78 & .001 & 16.701 & .001 \\
\hline SEIZURE & 1 & 7.62 & .006 & - & - & 5.30 & .021 \\
\hline BTHW'T & 1 & 5.10 & .024 & 6.69 & .010 & 5.39 & .020 \\
\hline APGAR5 & 1 & 47.34 & .001 & 38.21 & .001 & 31.09 & .001 \\
\hline SGA & 1 & 4.20 & .040 & 3.34 & .068 & 3.31 & .069 \\
\hline LBLOODP & 1 & 0.55 & .460 & - & - & 0.71 & .399 \\
\hline LTEMPF & 1 & 18.67 & .001 & 6.07 & .014 & 5.08 & .024 \\
\hline $\mathrm{PO} 2 \mathrm{FIO} 2$ & 1 & 15.53 & .001 & 1.50 & .221 & 1.78 & .182 \\
\hline LURINE & 1 & 12.91 & .001 & 24.88 & .001 & 23.06 & .001 \\
\hline LPLT & 1 & - & - & 13.91 & .001 & 12.39 & .001 \\
\hline HSODIUM & 1 & - & - & 24.70 & .001 & 24.28 & .001 \\
\hline HRESPR & 1 & - & - & 28.50 & .001 & 28.77 & .001 \\
\hline $\mathrm{HPCO} 2$ & 1 & - & - & 4.07 & .044 & 2.85 & .091 \\
\hline LGLUC & 1 & - & - & 6.71 & .010 & 6.84 & .009 \\
\hline HBLOODP & 1 & - & - & 0.02 & .862 & 0.04 & .840 \\
\hline
\end{tabular}

10.2.2.1 Relative Importance of the Variables in Each Model

When the units are different for each variable in the model, the interpretation and comparison of the beta coefficients and their associated odds ratios can be problematic. Thus, interpretation of the odds ratios can be more straightforward if the predictor values are all on 
the same scale. Because this is not the case with the SNAPPE-II and the MIRG predictors, the all variables (including the two dichotomous ones, seizure and SGA) were standardized before regressing them on the dependent. Table 13 shows the coefficients in terms of odds ratios $(\exp (B))$ for each of the standardized variables. These odds ratios were obtained for the models using the composite database because it allows for a direct comparison of the results from each of the models.

The results showed that the estimated coefficient values, or equivalently, the change in the odds corresponding to one unit change in the value of the standardized predictor variable, differed depending on the model used. For example, the variable $\mathrm{PO}_{2} \mathrm{FIO}_{2}$ has an odds ratio of 1.484 within the SNAPPE-II model and 1.136 within the MIRG model. This implies that the odds of living $(\mathrm{Y}=1)$ increases by $48 \%$ vs. $13 \%$ per unit increase in standardized $\mathrm{PO}_{2} \mathrm{FIO}_{2}$ respectively if the SNAPPE-II or the MIRG model is used for the prediction. Furthermore, if one were to use all 15 variables to predict the outcome, the estimated increase in odds of living for the baby would be $15 \%$.

As well, when a set of predictor variables are correlated (share variance) with each other, the standard errors of their estimates become highly inflated, resulting in rather unreliable estimates that can fluctuate greatly from sample to sample. The full correlation matrix for all 15 predictor variables is given in Appendix B. Hence, it could be argued that a better view of the importance of a predictor variable can be obtained by comparing the standardized betas obtained from a set of simple regressions of the outcome variables against each predictor itself. (See also Table 13).

Table 13. Odds Ratios in Standardized Units for All Variables in Each of the Models in 
the Composite Dataset.

\begin{tabular}{|c|c|c|c|c|c|c|}
\hline Z-Variable & $\begin{array}{c}\operatorname{Exp}(\mathrm{B}) \\
\text { SNAPPE-II }\end{array}$ & $\begin{array}{l}\operatorname{Exp}(B) \\
\text { MIRG }\end{array}$ & $\begin{array}{c}\operatorname{Exp}(\mathrm{B}) \\
\text { Composite }\end{array}$ & $\begin{array}{c}\text { Exp (B) } \\
\text { Simple } \\
\text { Regression }\end{array}$ & $\begin{array}{l}\text { Rank of Exp } \\
\text { (B) for } \\
\text { Composite }\end{array}$ & $\begin{array}{l}\text { Rank of Exp } \\
\text { (B) for } \\
\text { Simple } \\
\text { Regression }\end{array}$ \\
\hline Z-LBLOODP & 1.081 & - & 1.102 & 1.802 & 13 & 6 \\
\hline Z-LTEMPF & 1.353 & 1.198 & 1.182 & 1.734 & 8 & 7 \\
\hline Z-PO2FIO2 & 1.484 & 1.136 & 1.152 & 1.845 & 11 & 4 \\
\hline Z-LURINE & 1.440 & 1.710 & 1.679 & 1.814 & 2 & 5 \\
\hline Z-LSERUM & 1.376 & 1.501 & 1.469 & 1.863 & 4 & 3 \\
\hline Z-SEIZURE & 0.840 & - & 0.861 & 0.818 & 12 & 13 \\
\hline Z-BTHWT & 1.294 & 1.329 & 1.328 & 1.650 & 6 & 8 \\
\hline Z-APGAR5 & 1.606 & 1.543 & 1.491 & 1.965 & 3 & 2 \\
\hline Z-SGA & 0.884 & 0.890 & 0.890 & 0.849 & 14 & 15 \\
\hline Z-LPLT & - & 1.376 & 1.353 & 1.618 & 5 & 9 \\
\hline Z-HSODIUM & - & 0.698 & 0.698 & 0.624 & 7 & 10 \\
\hline Z-HRESPR & - & 1.700 & 1.713 & 2.287 & 1 & 1 \\
\hline Z-HPCO2 & - & 1.195 & 1.162 & 0.750 & 9 & 11 \\
\hline Z-LGLUC & - & 0.846 & 0.845 & 0.839 & 10 & 14 \\
\hline Z-HBLOODP & - & 1.013 & 0.980 & 1.245 & 15 & 12 \\
\hline
\end{tabular}

10.2.2.2 Interactions Among Model Variables

In order to examine the role of pairwise interactions among variables, the two-way interaction variables were examined and their contribution to the models analyzed. All 15 
variables were renamed $\mathrm{v} 1$ to $\mathrm{v} 15$ and 105 pairwise interactions from v1v2 to $\mathrm{v} 14 \mathrm{v} 15$ interactions were computed and added to the dataset as interaction variables.

For the SNAPPE-II model, the addition of the interaction variables significantly increased the model fit. The change in chi-square value was an increase of 73.78 significant at .05 (with 36 degrees of freedom). The Nagelkerke $\mathrm{R}^{2}$ and the $\mathrm{R}_{\mathrm{L}}^{2}$ also increased. For the MIRG model, the addition of the interaction variables also significantly increased the model fit. The change in the chi-square value was an increase of 136.7 significant at .05 (with 78 degrees of freedom). For the composite model, the addition of the interaction variables also led to a significant increase in the model fit. This increase in the chi-square value was 146.64 , significant at .05 (with 97 degrees of freedom). The Nagelkerke $\mathrm{R}^{2}$ and the $\mathrm{R}_{\mathrm{L}}^{2}$ also increased again.

None of the Wald tests of interaction variable coefficients were significant at the recommended Bonferroni-corrected alpha level of $.10 / 105=.001$. However, some of the Wald tests were significant at the .05 level. For the SNAPPE-II model, 6 out of the 36 tests of the interaction coefficients were significant; for the MIRG model, 10 out of 78 tests were significant; and for the composite model, 14 out of 105 tests were significant. A full list of these interaction terms is given in Appendix C.

The above analyses were also repeated by adding only the significant interaction variables to the corresponding models. For all models, the fit of the model substantially decreases when only those interaction variables are included in comparison to when all of the interaction variables are included. This indicates that for the most part, all of the interaction terms as a whole were important rather than some specific subset of them. 


\subsubsection{A Closer Look at an Interaction Variable}

In order to better understand the interaction variables, further analyses were performed on one interaction variable: "lowest blood pressure $\mathrm{x}$ lowest temperature" in the SNAPPE-II model. The variable Lowest temperature had a significant partial coefficient estimates $(\exp (B))$ at .05 alpha level but the variable lowest blood pressure did not. However, the interaction variable "lowest blood pressure $\mathrm{x}$ lowest temperature" had a significant estimated partial beta coefficient, that is with all other interaction terms added, it was significant at $\mathrm{p}<.015$. In the composite dataset, the cases were divided into three groups according to their values (lowest temperature: $1<95,96<=2=>95,3=>96$ and lowest blood pressure: $1<20,29=<2=>20,3=>30$ ). The predicted non-mortality probabilities for the variable lowest blood pressure were then plotted as a function of lowest temperature in Figure 1. The lines in the figure are not parallel, indicating an interaction. The estimate of the change in the probability of non-mortality as a case moves from one category of lowest blood pressure to another is the vertical distance between the two lines (Hosmer et al., 2000). This difference is not the same across all values of lowest temperature, indicating that the relationship between temperature and survival for babies depends on the value of blood pressure. That is, the probability of life is differentially enhanced with increases in lowest blood pressure at each level of lowest temperature. This implies that temperature modifies the effect of blood pressure and hence the estimate of the odds ratio related to blood pressure can only be interpreted in terms of temperature. 


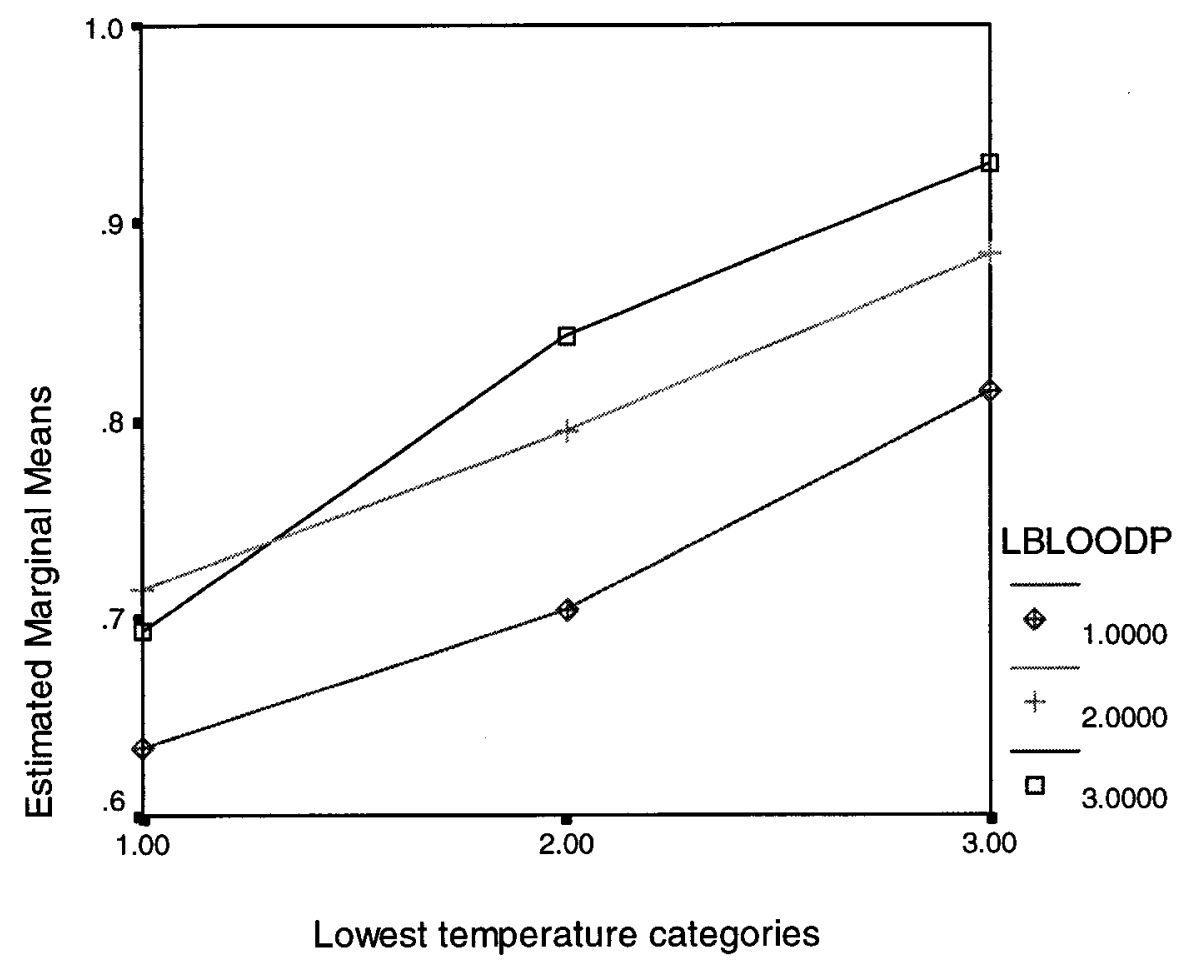

Figure 1. Estimated Marginal Means of Predicted Probability for Lowest Blood Pressure as a Function of Lowest Temperature in 3 Categories in Ascending Order.

10.3 Examining the Predictiveness of the Models

\subsubsection{Classification Cutoff Value}

A classification cutoff value is used by SPSS to specify the cutoff value for predicting outcomes. This value is used as the decision threshold used to generate classification tables for models. The classification tables compare the number of correct versus incorrect predictions. The default value for the classification cutoff in SPSS is 0.5. This value indicates that if the conditional probability of life (i.e., $\mathrm{Y}=1$ ) predicted by the logistic regression exceeds 0.5 , the model will predict life and if the conditional probability of life is less than 0.5 , the model will predict death. In order to find the best cutoff point for a dataset using a particular model, several cutoff values must be examined to find the cutoff point at which the proportion of 
incorrect prediction is the lowest. Note, though, both that correct predictions of death and of life are associated with different underlying prevalence rates and that different costs or consequences are likely to be associated with different incorrect predictions of death and of life.

Table 14 shows values of the different cutoff points and their associated correct death (sensitivity) and life (specificity) predictions. The results show that for each gain in the correct prediction of death, there is a loss in the correct prediction of life. The decision threshold for any particular clinical setting may be a function of the utilities associated with the predictions. If the utility of a correct positive prediction of life is very high, then the best choice may be to predict that all babies will live. In that case, there will be no need for a predictive model with a set of independent variables, and the clinician would err $10 \%$ of the time (given the survival rate of $90 \%$ ). However, if the prospect that some sick patients with no, or very little, chance of survival will be treated aggressively is regarded as engendering a high utility cost to incorrect positive prediction of life, then a more lenient decision threshold would be required. If the clinician chooses to treat, or aggressively treat, all babies regardless of the probability of outcome, then no decision threshold is needed. The most likely scenario however, is one where the decision threshold is modified for different cases, or groups of cases, depending on the severity of the underlying disease. 
Table 14. Cutoff Values and Associated Percent Predictions for SNAPPE-II variables in Composite Dataset.

\begin{tabular}{llcc}
\hline Cutoff value & $\begin{array}{l}\% \text { correct death } \\
\text { prediction }(\mathrm{N}=247)\end{array}$ & $\begin{array}{c}\text { \% correct life } \\
\text { prediction } \\
(\mathrm{N}=2155)\end{array}$ & $\begin{array}{c}\% \text { total correct prediction } \\
(\mathrm{N}=2402)\end{array}$ \\
\hline
\end{tabular}

SNAPPE-II model

\begin{tabular}{|c|c|c|c|}
\hline \multirow[b]{2}{*}{0.95} & \multirow[b]{2}{*}{89.5} & \multirow[b]{2}{*}{47.5} & \multirow[b]{2}{*}{51.8} \\
\hline & & & \\
\hline 0.90 & 70.4 & 74.4 & 74.0 \\
\hline 0.85 & 59.9 & 85.7 & 83.1 \\
\hline 0.80 & 50.2 & 91.0 & 86.8 \\
\hline 0.70 & 31.2 & 95.8 & 89.2 \\
\hline 0.60 & 18.2 & 98.0 & 89.8 \\
\hline 0.50 & 14.6 & 99.2 & 90.5 \\
\hline 0.40 & 12.6 & 99.5 & 90.6 \\
\hline 0.30 & 4.9 & 99.8 & 90.0 \\
\hline 0.20 & 2.4 & 99.9 & 89.8 \\
\hline \multirow[t]{2}{*}{0.10} & 0.4 & 100.0 & 89.7 \\
\hline & \multicolumn{3}{|c|}{ MIRG model } \\
\hline 0.95 & 90.3 & 56.4 & 59.9 \\
\hline 0.90 & 76.1 & 76.7 & 76.6 \\
\hline 0.85 & 64.4 & 85.4 & 83.2 \\
\hline 0.80 & 56.3 & 90.1 & 86.6 \\
\hline 0.70 & 40.1 & 94.9 & 89.3 \\
\hline 0.60 & 30.4 & 97.4 & 90.5 \\
\hline 0.50 & 21.9 & 98.8 & 90.0 \\
\hline 0.40 & 14.2 & 99.5 & 90.8 \\
\hline 0.30 & 9.3 & 99.8 & 90.5 \\
\hline 0.20 & 5.7 & 100.0 & 90.3 \\
\hline 0.10 & 2.0 & 100.0 & 89.9 \\
\hline
\end{tabular}




\section{Composite model}

\begin{tabular}{llll}
0.95 & 91.1 & 56.8 & 60.4 \\
0.90 & 76.5 & 77.0 & 76.9 \\
0.85 & 64.8 & 85.4 & 83.3 \\
0.80 & 55.90 & 90.0 & 86.5 \\
0.70 & 40.5 & 94.6 & 89.0 \\
0.60 & 30.8 & 97.5 & 90.7 \\
0.50 & 22.7 & 98.8 & 91.0 \\
0.40 & 14.2 & 99.5 & 90.7 \\
0.30 & 9.3 & 99.8 & 90.5 \\
0.20 & 5.7 & 99.9 & 90.2 \\
0.10 & 1.6 & 100.0 & 89.9 \\
\hline
\end{tabular}

\subsubsection{Receiver Operating Characteristic Analysis}

In order to examine and compare the predictive ability of models, an ROC analysis was carried out using the composite dataset $(\mathrm{N}=2,402)$. AN ROC analysis compares the predictive abilities of each model across a range of classification cutoff values. The ROC curve represents a plot of sensitivity (hit) against 1 - specificity (false alarm) at each of these cutoff values. These are calculated from proportions of observed death outcomes that were correctly predicted and observed life outcomes that were incorrectly predicted, respectively.

The ROC curve shows how the two probabilities vary together as the decision threshold is changed. The area under the curve assesses the models' ability to discriminate between the two outcome categories. Table 15 shows the area under the ROC curve for various models with and without their interaction terms (note that models were fit separately 
to the low birth weight cases to derive their predictions. All of these areas were derived using the non-parametric ROC procedure specified by Hanley and McNeil (1982) For the MIRG model, the area under the ROC curve was .8323 and for the SNAPPE-II model it was .7950 (see Figure 2).

Table 15. Comparison of the Areas Under the ROC Curve for Models With and Without their Interaction Terms.

\begin{tabular}{lc}
\hline Model & Area Under the ROC \\
\hline SNAPPE-II & .7950 \\
SNAPPE-II with Interactions & .8336 \\
MIRG & .8323 \\
MIRG with Interactions & .8661 \\
Composite & .8391 \\
Composite with Interactions & .8777 \\
Low Birth Weight SNAPPE-II & .7724 \\
Low Birth Weight MIRG & .8035 \\
Low Birth Weight Composite & .8126 \\
\hline
\end{tabular}

A statistical comparison of the areas under the curve (Hanley \& McNeil, 1982) for the two SNAPPE-II and MIRG models without the interaction terms showed the difference between them is significant $(Z=3.726, \mathrm{p}<.05$.$) , indicating that the MIRG model's predictive$ ability is somewhat superior to that of the SNAPPE-II model. As well, although methods are available (Swets et al., 2000) for deriving the optimal cutoff points for decision making purposes using base rates and utility information, this was not attempted here. 


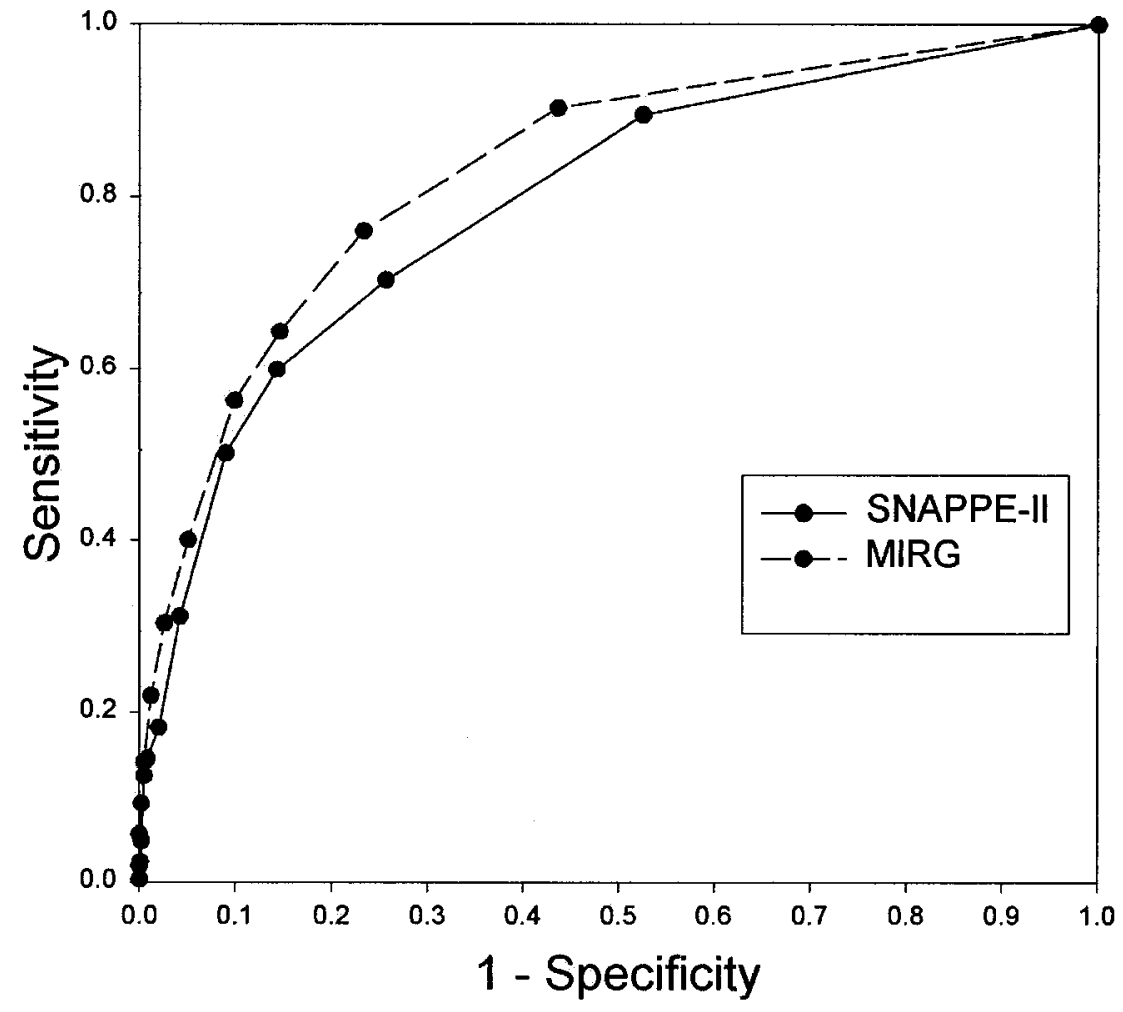

Figure 2. ROC Curve for Discriminative Ability of MIRG vs. the SNAPPE-II Model 


\section{INTERVIEW RESULTS}

This section outlines the results of the exploratory interviews with the neonatologists. The comments and answers of neonatologists to the questions asked during the interviews are sorted into themes in the following section.

\subsection{Descriptive Information}

The Ottawa Hospital has two campuses with NICUs, the General campus and the Civic campus. In addition, the Children Hospital of Eastern Ontario (CHEO) has a NICU which accepts "transfers" from the other two NICUs in Ottawa and from NICUs of other hospitals within the Province of Ontario. The NICU in CHEO receives either very sick infants requiring special care that cannot be offered in a regular hospital NICU or infants requiring surgery. The seven neonatologists interviewed had their offices and primary practice at one centre, but also worked at one or the other two centres in the city. After the second interview, it became evident that the types of health problems primarily dealt with at $\mathrm{CHEO}$ were more severe than the other two centres, and required special management such as surgery.

All neonatologists were familiar with the concept of DSS through their knowledge of the work of their colleague at CHEO (R. Walker). The participants' experience in neonatology ranged from 1 to 30 years. The self ratings as computer users ranged from novice to expert with one novice, two experts, and the rest falling between these two extremes. All but one of the participants used at least three of the software packages that could be regarded as examples of the types of software that neonatologists might be using. Other software packages identified 
were Microsoft Excel, Photoshop, and Media Blair. For other uses of the computer see Table 16.

Table 16. Participants' Reasons for Computer Use

\begin{tabular}{lccccl}
\hline Email & Study & $\begin{array}{c}\text { Search } \\
\text { info }\end{array}$ & News & Entertain. & Other \\
\hline & & & & $\begin{array}{l}\text { Teaching, } \\
\text { Administration, } \\
\text { Personal Finances, } \\
\text { Research, Preparing } \\
\text { Presentations }\end{array}$ \\
\hline
\end{tabular}

After the second interview, it also became clear that neonatologists in different centres might be interested in different variables because of the nature of the centres where they worked. The NICU at CHEO receives infants transported from other NICUs. No infants are born at $\mathrm{CHEO}$. According to one interviewee, some variables become less important at the time of admission to CHEO. This is because often specific problems have been dealt with and corrected at the NICU or at the hospital where the infant came from. For example, under certain circumstances, low glucose levels and temperature of the newborn are important factors within minutes of a baby's birth. They are corrected shortly after birth, often requiring simple procedures such as wrapping the baby in a blanket. Alternatively, the problem is corrected during transport of the baby to CHEO. Once the baby is at the NICU in CHEO, the previously noted problems of low glucose levels and low temperature are no longer important factors in determining a baby's prognosis, presumably because the infant has been warmed up and the glucose levels have already been corrected. Those same variables, however, 
might be important to the neonatologist who is either present at the baby's birth or sees the baby shortly after birth in NICU. Hence, such context-dependent differences in the importance of the variables to the neonatologists should be taken into account when interpreting the results of this section.

The first interview was conducted at $\mathrm{CHEO}$, and followed by a short tour of the NICU facility where the possible locations for a DSS were pointed out. The NICU at CHEO is made up of several rooms where the babies are monitored. There is also at least one area at a nursing station where several beds and various machines are housed and used for babies requiring constant monitoring. The nursing stations are equipped with computers. The physical space seemed to give ample opportunity for installation and use of a DSS.

\subsection{General Views About the Value of a Prognostic DSS in a NICU}

All neonatologists agreed that they would use a prognostic DSS in the NICU but some expressed certain reservations about its value in a NICU. All believed that a DSS would be useful as one tool among others, such as the option of looking through NICU files for information or consulting a fellow neonatologist. All judged themselves likely to use the system. One interviewee expressed some reservations, though still believed he would use it if time permitted, saying

“... if the machine and data were available in the clinical area so that sometime during my day's work I could sit down and see what the computer suggests about a new patient or a specific patient program, I would [use the prognostic DSS]".

All stated that they would mainly rely on their own judgment, but use the DSS when faced with uncommon or difficult clinical cases. As to the reasons for using the DSS, six out of 
seven interviewees agreed that the prognostic DSS would be particularly useful for counseling parents, while one neonatologist believed he would use the DSS either out of "interest" or as a memory aid to "refresh memory", but not for help with counseling parents. This neonatologist's reservations were concerned with the use and applications of statistical information in general. Although all considered themselves likely to use the DSS, most expressed some degree of reservation, in general, about the use of DSS. Two had opposing views, in that one was suspicious about using any statistical measure for decision making and the other believed statistical measures were completely reliable. One made statements such as

"I always have concerns about applying statistical information in a specific individual patient care situation and I always caution parents about that... I'm indeed reluctant and usually refuse to give specific predictions [based on statistics] because I don't think it applies to their particular child. Each baby is different, each case is specific in terms of its severity and probability of survival or long term outcome and I really don't feel it's fair to hazard what would be nothing more than a guess"; and "over the years, I tend to be optimistic in most cases, and as I say to families it usually serves me well because in my past experience [cases are] more favorable than I feared".

Another neonatologist had an opposite view stating

"having that prognosticating [help from] some sort of simulated computer program telling us that if at 48 hours the baby's status is such and such, then this baby statistically has a potential chance of making it without any injury or an intact brain [sic], is this much. And for us to use this information to counsel parents is good, and I think it is quite useful at least in this specialty...for helping [the parents] understand that given all the [information from the] database into the computer, [the] computer actually generates a probability of mortality of your child [that] is almost certain"; and "For example, again, a 24 weeker weighing $500 \mathrm{~g}$, all in the viability line, and the parents choose to treat the baby, we will have then the ability to tell them within 24 hours the chance of this baby's survival. And if the chance of survival is only less than $5 \%$, for this baby who is extremely sick, more or less than $1 \%$, and the chances of death being $99 \%$, then it would be extremely beneficial to say whether we should carry our efforts at all". 
These statements from two different participants show very different positions. The positions may reflect differences in decision thresholds or in the degree of confidence placed in statistics. It should be noted that the latter statements came from a participant who considered himself an expert computer user, who currently was using computers for teaching and research in addition to all the purposes suggested in the Participant Information sheet. The former statements were made by a participant who considered himself a novice computer user, who was currently using computers only for sending or receiving emails.

Other neonatologists expressed views that fell between these two extremes. They expressed some degree of reservation due to concerns about the source of information, that is in the database itself, such as the potential for error in terms of data entry or data gathering by humans or machines, and the resources available to the hospital NICUs who participated in collecting information for the database. For example, one neonatologist stated

“...you may have a first- or second- year resident in pediatrics, not in neonatology, attending the delivery of a baby whose skill level may not be up to standard yet because they're in training. And they may, as a result of inadequate resuscitation, cause many abnormalities in these parameters. So, ... in my situation, when I enter the data for a baby I have personally managed, I want to know what correlation there is with mortality with my situation, not against a first-year resident in xxx who is part of your database. The relevance of that will become less so with the more data you collect, and if you have data across Canada over 5 years, for example, the outliers will become less and less so".

Another neonatologist expressed concerns, in response to the question of whether he saw himself likely to use the prognostic DSS, by saying

"It all depends on where the information comes from and how up-to-date the information is. Things change from year to year and week to week. I wouldn't use a system that was based on [a small database], if it's going to be anything, 
it's going to have to be ...not [even specific to the country], I don't think country is specific enough. No, every hospital is different, every group is different. I'm not sure that we can always extrapolate from one group and one area to another group and another area. Yes, there are trends, but I'm not sure...I think that hospital-specific data are far more important than countryspecific data". And "Our expertise is our expertise here. The expertise here isn't the expertise in $\mathrm{xxx}, \ldots$, we can't offer an outcome that is wonderful in xxx, we can't offer it here all the time because we may not have [the resources available]. So, yes I'll use [the prognostic DSS], but based on those limitations. If it's not appropriate to what's happening here, it may not be as important'.

Almost all had concerns about the size of the database. They referred to the adequacy of the statistics used by the DSS in terms of the available "statistical power" due to the sample size used to generate results. They expressed their concerns in terms of level of trust they were able to place in the results of a DSS.

Regarding interacting with a DSS, all reported that using the DSS in a NICU or their office would pose no problems. They all stated that a "user-friendly" interface would take away any or all concerns about their interactions with the DSS. Some suggestions were provided such as the use of menu-driven options, pen-lights, touch-screens, and keyboards.

\subsection{Views About the Accuracy, Reliability and Trustworthiness of DSS Output}

The participants' opinions about accuracy, reliability, and trust in a DSS output varied.

Results are shown in Table 17. The scores for accuracy and reliability ranged from 40 to 90 . Scores about trust in information gained from a DSS output ranged from 40 to 100 . The question about accuracy was intended to probe neonatologists' thinking on two dimensions. First, whether they considered the accuracy of the data in databases which are subject to human or machine error, second, whether they considered the accuracy (sensitivity) of the reported test results. 
Table 17. Participants' Rankings for Accuracy, Reliability, and Trust of a Prognostic DSS Output

\begin{tabular}{cccc}
\hline Questions & Accuracy & Reliability & Trust \\
\hline Range & $40-90$ & $40-90$ & $40-100$ \\
Mean & 79 & 70 & 77 \\
\hline
\end{tabular}

The reasons given for their choices for the accuracy question varied. At one extreme was a high degree of skepticism, at the other, a high degree of confidence in the accuracy of an output from a DSS. For example, one participant said

"If the report would be based on experience with 10 patients, I would not consider that very valid. Ten cases is not a large experience... [even if cases were chosen from among a $20-30 \mathrm{~K}$ database] I would still be skeptical".

An opposite view was stated from another neonatologist

"Knowing the last 10 cases across the board in Canada and [knowing that] this is what the outcome is, it actually helps you to know that for example $60 \%$ of them actually did die, even though maybe I was leaning toward actually doing something with the baby. This will help me counsel the parents as well, [telling them] that this is not just my hand, looking across the board in the country, internationally, let's say using the xxx database which considers 160 centres all across the world, across all continents, then we can compare our colleagues across the world in terms of what is happening in Australia or what is happening in Europe".

The two statements above seem not to consider the potential accuracy of the DSS output in terms of either sensitivity or erroneously entered data. Perhaps there may have been a better way of formulating the question to be able to get the purpose of the question across 
differently. However, other neonatologists did seem to consider these issues. For example, a neonatologist's answer to the question was

“[I say] 85\% accurate. Ok, I'm throwing numbers out. [I don't know] what variables you're putting in, you tell me what you're putting in, and I'll tell you if I think it's useful."

And other comments were

“[can't accept accuracy] until I know what the system is, and who's putting it in, and how many errors there are, and so on ... If you can guarantee that what goes in is accurate, what comes out will be accurate. I'll need to know how accurate the information going in is. I'll need reassurance on that point, then [I might accept] that it is accurate".

"In terms of accuracy? For example, blood pressure is really what it says it was? Well, it's not a hundred [percent] for sure, because it depends on who enters the information, right? So, I'd say probably 90 percent. There's always entering data issues".

The question regarding the reliability of the output was intended to identify the participant's considerations of the reliability of a DSS functioning such as its ability to match similar cases correctly and consistently. It was also meant to examine whether the participants were concerned, for example, about the reliability of the software's algorithm.

The question about trusting the output was intended to examine the participant's overall attitude toward using information provided by a DSS. It is possible that one would take into consideration the probability of data entry errors and the probability of false positives, and be able to judge the overall information in the light of those probabilities, and yet be reluctant to trust and use the information from a computer. 
Assuming that the purpose of the questions were recognized, most of the participants seemed to believe that the accuracy of a DSS output was the same as, or related to, the reliability of the output. And several believed that reliability and accuracy were the same as the trustworthiness of the output. Three of the participants said that they would consider relying on and trusting the output but only after testing the system personally by using it for an extended period of time.

The statements showed some of the reasons given for the chosen accuracy levels of information gained from a DSS (ranging from 40 to 90). Most participants believed the output would be accurate, given certain caveats, such as the use of appropriate variables in the predictive model and the accuracy of data (values for the variables) entered. The participant with the least amount of computer use who considered himself a novice gave the lowest ratings for all three questions yet with the caveat "assuming that [data source] was from a comparable population managed by comparable individuals". One of the participants who considered himself an expert computer user seemed to be more likely to trust the information, saying

"The analysis is done with data collected and there is a data which is given to me, just a raw data, then I'm going to trust it exactly as it is, as long as there is quality assurance attached to that collection of data pieces, then I have no reason not to trust the data. Because there is no reason why people should give wrong information, so then I would trust it $100 \%$."

\subsection{Opinions About the Effects of Information From a DSS on Participants' Confidence}

The elicitation of opinions concerning the effects of the information provided by a DSS on participants' confidence in their own judgments was obtained in three ways. First, 
participants were asked a generic question about the change in confidence. All believed that in general, the DSS output would not affect their confidence. The second and the third questions were more specific. They were asked about situations where a DSS output might either agree or disagree with their own a priori hypothesis. All believed that if the DSS output agreed with them, they would have increased confidence. Four out of seven believed that if the DSS's output disagreed with their own hypothesis, they would rethink their judgment. Two would rule in favor of their own judgement, and one said that if he believed a baby would live and the DSS disagreed, he would redouble efforts and try to find ways to increase the baby's chances of survival.

\subsection{Opinions About the Variables}

Neonatologists were first asked to give the three variables that they regarded as being the most important for prognosis. Then, they were presented with a list of the 15 variables and asked to rank order them in terms of importance. With respect to the first task, they all believed that the field of neonatology was too large and there were too many variables that could potentially affect the outcome, making it difficult to choose only three variables that would apply to all cases. Table 18 lists the three most important variables chosen by each neonatologist. Five out of seven agreed that gestation age was among the three most important variables, and three out of seven agreed that birth weight was among the three most important variables. 
Table 18. The Three Most Important Variables for Prognosis According to Participants

\begin{tabular}{lccc}
\hline Participant & Variable 1 & Variable 2 & Variable 3 \\
\hline 1 & Gestation age & Birth weight & $\begin{array}{c}\text { Some measure of disease } \\
\text { severity- disease specific }\end{array}$ \\
2 & Gestation age & Need for ventilation & Medication \\
4 & Gestation age & Birth weight & Need for ventilation \\
5 & Gestation age & Birth weight & Gender/race \\
6 & Gestation age & Ventilation Status & Lowest blood pressure \\
& Apgar at 10 & Lowest mean blood & pressure \\
7 & Diagnosis & A severity-of-illness & Serum Ph \\
\hline
\end{tabular}

In the rank-ordering task, variables from the MIRG and the SNAPPE-II were combined, resulting in 15 variables. Six out of seven neonatologists agreed that the variable birth weight was the most important variable in making prognosis, and one neonatologist ranked it as the second most important variable. Table 19 shows the mean and the range of ranks assigned to each variable. 
Table 19. Ranking Statistics For the $15 \mathrm{MIRG}$ and SNAPPE-II Variables (Where the Most Important Rank = 1).

\begin{tabular}{|c|c|c|c|c|}
\hline Variable & Range & Lowest & Highest & Mean \\
\hline BIRTHWT & 1 & 1 & 2 & 1.1 \\
\hline LBLOODP & 4 & 2 & 6 & 3.3 \\
\hline SGA & 6 & 2 & 8 & 4.4 \\
\hline SERUM & 10 & 1 & 11 & 5.0 \\
\hline APGAR5 & 12 & 1 & 13 & 5.5 \\
\hline PO2FIO2R & 10 & 2 & 12 & 6.3 \\
\hline LTEMP & 10 & 3 & 13 & 7.0 \\
\hline LURINE & 9 & 2 & 11 & 7.1 \\
\hline $\mathrm{PCO} 2$ & 10 & 3 & 13 & 7.3 \\
\hline SEIZURE & 14 & 1 & 15 & 8.4 \\
\hline LGLUC & 11 & 3 & 14 & 8.4 \\
\hline RESPRATE & 11 & 4 & 15 & 9.0 \\
\hline SODIUM & 10 & 4 & 14 & 9.1 \\
\hline LPLTL & 13 & 2 & 15 & 9.8 \\
\hline HBLOODP & 9 & 6 & 15 & 10.7 \\
\hline
\end{tabular}

Table 18 and 19 do not show very much internal reliability among the physicians regarding the importance of the variables. The average of the 21 pairwise Spearman rank correlation coefficients between the seven rankings is $r_{s}=.332$. Comparing the mean of these rankings with the simple and partial beta coefficient rankings from Table 13, Spearman rank 
correlations between these means and each of these statistically based rankings were obtained (see Table 20). The results show that the neonatologists' average rankings of the variables do not correlate significantly with rankings based on either simple or partial B's and there is a negative correlation between rankings given by neonatologists and the rankings obtained from the partial beta coefficients.

Table 20. Spearman Correlation Coefficients for the 15 Composite Variable-Rankings by Clinicians, Simple Regressions, and Partial Regressions.

\begin{tabular}{|c|c|c|c|c|c|}
\hline & & & Clinicians & Simple B & Partial B \\
\hline \multirow[t]{9}{*}{ Spearman's rho } & Clinicians & $\begin{array}{l}\text { Correlation } \\
\text { Coefficient }\end{array}$ & 1.000 & .252 & -.025 \\
\hline & & Sig. (2-tailed) & . & .365 & .929 \\
\hline & & & 15 & 15 & 15 \\
\hline & Simple B & $\begin{array}{l}\text { Correlation } \\
\text { Coefficient }\end{array}$ & .252 & 1.000 & $.696^{*}$ \\
\hline & & Sig. (2-tailed) & .365 & . & .004 \\
\hline & & & 15 & 15 & 15 \\
\hline & Partial B & $\begin{array}{l}\text { Correlation } \\
\text { Coefficient }\end{array}$ & -.025 & $.696^{*}=$ & 1.000 \\
\hline & & Sig. (2-tailed) & .929 & .004 & \\
\hline & & & 15 & 15 & 15 \\
\hline
\end{tabular}

${ }^{* *}$. Correlation is significant at the .01 level (2-tailed). 


\subsection{Additional Concerns}

\subsubsection{Concerns Regarding Lack of Trend Information}

Five out of seven neonatologists expressed concerns about lack of "trend"

information. For example,

"It is not the data in the first 12 hours of life that is going to determine the outcome." And "trends [are needed because] as the information goes in [sic], you can see the trends, gives you a chance to look at whether or not things are changing]. Also "I need to know different scores on different days".

\subsubsection{Concerns Regarding Lack of Gestation Age as a Variable}

All participants expressed concerns about the lack of gestation age in the models. Some agreed that SGA could be useful if the exact percentile were provided as opposed to a broad classification into yes and no categories, which is the case now. For example, a participant commented

"you're treating a 26-week gestation baby the same way as you're treating a 39week gestation period? Now, at 10 percentile 26-week gestation baby inherently, to me, would be at much greater risk of mortality than a 10th percentile 38-week baby--which is a fault of this." And "if you don't have the gestational age for my baby, that you're giving back the scenario for a 27-week baby when I've punched in the date of birth of the 28-week baby. There's no connection. How would I use your information? It's not appropriate for my situation"

Similar concerns were expressed by all participants.

\subsubsection{Concerns About Dated Information and the Lack of Centre-Specific Data}

There were concerns about the prognostic DSS output not being centre specific. While a participant agreed that it was better to have a large database than a small centre-specific database, concerns seemed to show the dependence of prognoses on hospital resources. One 
participant commented "I can only know what my ability is-and that would relate to what I can do here". There were also concerns about dated information and the rapid of rate of change in resources and practice. For example, one participant said

"I need to know over how much time the information has been collected: whether over the last six months or previous year or 5 years or 10 years, because things [change] in some areas and not other areas”

However, it seemed that among the different options, the option of having a larger database as source data for a DSS would be preferred by neonatologists. 


\section{DISCUSSION}

The results of this study suggest that interviewed neonatologists view a prognostic DSS as a useful tool in NICU. Many have reservations about the accuracy of a DSS output. However, they see themselves likely to use the DSS as one of the available supportive tools. Many believe they would be more likely to trust the system and use it in their decision making if they fully understood how it works and where its data originates from. All of the interviewees expressed concerns about the variables that were used to predict mortality. They did not agree on what the three most important variables were but the lists of important variables they gave overlapped. The neonatologists also did not agree on the degree of importance of each of the variables for prognostic decision making. The variables rankings, with the exception of birth weight being the most important variable, were inconsistent. There are several possible explanations for the different rankings. It could be due to the low number of interviews. It could also be due to the difference in years of experience of the participants or the differences in the NICU populations they worked with. Regardless of the reason for the inconsistent variable ranking, it is important to recognize that a prognostic DSS that proposes to use one model to predict neonatal mortality outcome, will potentially be used by different centres, which likely serve different populations. It may be reasonable to consider use of different predictive models for different NICUs.

If the prognostic DSS is to be used only by similar centres, then additional studies should be undertaken to determine the requirements of a group of similar centres. When the requirements are defined, a proper predictive model can be implemented. If, on the other 
hand, the prognostic DSS is to be used by different centres, then additional studies are need to improve the predictive efficiency of the proposed model for each of these contexts.

Results of interviews also indicated that neonatologists require more information than that imparted by the set of variables included in the SNAPPE-II and MIRG models. They want other variables to be considered, including severity of illness scores, diagnosis, accounts of pregnancy and interventions, as well as variables that convey trends and development of physiological pathology over time.

Based on the results of the interviews, it was concluded that building a neonatal mortality model needs the guidance of theory and subject matter experts, in addition to rigorous statistical testing.

\subsection{Database analyses}

Quantitative analyses of the SNAPPE-II and MIRG model variables were also undertaken using mostly logistic regression methods. Given the large sample size in the datasets, it was reasonable to assume that after removing the outliers from the dataset, the data could be considered reliable and useful for model comparison.

In logistic regression, the likelihood values are compared to initial likelihood values when the model assumes that the coefficients are equal to zero. The chi-square test is a test of the likelihood that an outcome is observed given the values of the model variables. The larger the chi-square value, the greater the likelihood that the values of the independent variables have produced the observed outcome. The results showed that both models increased the likelihood of observing the outcomes in a statistically significant way. The chi-square value is 
larger for the MIRG model than for the SNAPPE-II model and it is largest for the composite model which contains all possible variables. This suggests that the addition of more variables increases the likelihood that the values of these variables can account for the outcomes, indicating that the rule of parsimony does not apply. When building such models, decisions need to be made regarding the point at which any potential increases in predictive ability are offset by potential difficulties in collecting the necessary data.

The rate of mortality in all datasets was about $10 \%$. This percentage was considered the prior probability of a death outcome for all datasets. In other words, one could predict that all of the babies in the database would live and be wrong $10 \%$ of the time. This meant that any predictive model used should likely do better than this probability. However, there are two types of errors that can occur with these predictive models. One is the misclassification of death outcomes (i.e., misses) and the other is the misclassification of life outcomes (false positives). Each error carries a cost. The utility of these costs are subjective. If the purpose of a model is economical, for example, if the model is used to decide the best course of action to reduce NICU resource expenditures, a wrongful prediction of death will have a lesser cost than a wrongful prediction of survival. This assumes the decision threshold is such that babies with a high probability of death are not treated. If hospital resources are not a concern, it is reasonable to suppose that incorrectly predicting life for a patient who will eventually die is less costly than incorrectly predicting death for a patient who will live.

It follows that the predictive ability of models with respect to one type of error can only be assessed with knowledge of the prevalence of errors of the other type as well. This is exactly the type of knowledge provided by an ROC analysis. With respect to the ROC analysis 
performed here, the MIRG model was shown to be better than the SNAPPE-II model at reducing classification mistakes of both types across the whole range of possible decision thresholds. Hence, in general, the MIRG model with more variables is statistically better than the SNAPPE-II model with fewer variables. It could be that the MIRG model is more of a "proper" model than the SNAPPE-II model. That is, the MIRG model might be better supported by clinical theory than the SNAPPE-II model.

Earlier, it was noted that the Canadian NICU database contained more information about babies with a higher risk of mortality, presumably because more clinical assessments were necessary for the sicker babies. This may imply that the datasets obtained here might over-estimate the probability of death for a normal NICU population. The interview results with neonatologists suggested that the neonatologists are less likely to use a prognostic DSS for the less sick population of a NICU. They are more likely to use a prognostic DSS when the patient is very sick, or has an unusual condition.

Results of interviews with neonatologists indicated that babies weighing less than $1000 \mathrm{~g}$ have a smaller chance of survival than larger babies, no matter what is wrong with them. A dataset consisting of cases with birth weights less than or equal to $1000 \mathrm{~g}$ was therefore examined for the prognostic ability of the models with this sample population. The area under the ROC curve for this dataset was smaller than the other two datasets and did not show improved overall prognostic efficiency for all models when the low birth weight data was used as the source data. The results suggest that increasing the prevalence of death outcomes, that is increasing the death to life ratio in the dataset, changes the predictive ability of the models but not necessarily in the expected direction. This is an unexpected finding given that 
sensitivity is closely tied to prevalence. The premise that the prognostic DSS should ideally be based on a database that is representative of the population the system is used for, in order to improve the predictive ability of the models, is not supported by these results. Furthermore, they lead to the supposition that using skewed datasets for model testing, and for the training of ANN to impute missing values, may not be misplaced.

Among the variables themselves, the highest respiratory rate had the highest statistical rank, suggesting the highest respiratory rate contributes the most to the prediction of the outcome for this dataset. However, birth weight was the variable ranked most important by the neonatologists. Overall, statistically driven ranks did not correlate significantly with the ranks given by neonatologists. That is, the statistical importance of the variables did not necessarily correlate with those the neonatologists considered to be important. One exception to this was the highest blood pressure, which all experts agreed was not a particularly important variable to consider for prognosis and also statistically ranked the lowest. In general, this discrepancy between the physician and statistical ranking of the variables is an important result that deserves further examination and study. Unfortunately, it cannot be determined with certainty here whether this result occurs because the physicians are privy to some practical insights about the workings of these variables that the statistical model does not have or vice versa. However, one finding that might shed some light on this issue is the fact that the physicians' rankings are correlated more positively with the betas from the simple regression which indicates that physicians might not be that sensitive to variable multicollinearity (i.e. redundancy). 
The results from interviews with the neonatologists suggested that most of the variables presented to them from the SNAPPE-II and the MIRG models were interdependent. The variables can and often do interact with each other. A low birth weight for example is mostly linked to the gestational age of the baby, which is associated with, or can lead to the presence of, seizures, which might affect urine output in a domino effect. Although only some of the possible interactions among the 15 variables were examined in this thesis, all paired interactions were analyzed. These results suggested that the addition of the interaction variables as a whole adds to the likelihood that the variables and their values are related to the outcome. However, few of the interaction variables contributed significantly to the model by themselves. This suggests that it is the set of the interaction variables, as a whole, that improves the performance of the models predicting outcome probabilities. Without further knowledge of higher order interactions among variables, such as multiple interactions, it is difficult to interpret these results. It can however be deduced that the more variables that are added to the models the better they perform.

\subsection{Interview analyses}

All of the neonatologists interviewed expressed a desire to have access to more and perhaps all, relevant information. Moreover, relevant information seemed to depend on the underlying disease, and the severity of illness. It includes the history of patient management along with birthing events such as mode of delivery and required interventions. Other factors include the medication, the mother's health status, and according to at least one interviewee even the father's health history including race and country of origin. 
All interviewees thought that the nature of their field requires them to have a great deal of information available. Not only would this information be valuable to them when making a prognosis about their patients, but also when they read and interpret decision-tool outputs about similar cases. Recall that in order to make a decision, clinicians are likely to rely on their past experiences. The probability that a clinician assigns to an outcome is based on his or her past experiences with similar patients. To make a prognosis, the clinician also needs to know, or calculate, the posterior probability $\mathrm{P}$ (outcome / evidence). The posterior probability is often a source of confusion, and difficult to obtain. The CBR-ANN hybrid DSS examined in this thesis does not provide clinicians with the posterior probability regarding a patient's outcome, however, it has the advantage of providing information about similar cases. To interpret similarities, the neonatologists maintained that they needed to have information about the health of the reported similar cases above the model variables reported by the DSS, such as diagnosis and health history since birth, trends and progress of health conditions over time, gestation age, race, gender, and even a case's parents' age and state of health, among others. These affect the prior probabilities of outcome for each baby reported similar by the DSS, without which the provided similarity information are difficult, if not impossible, to interpret. However, the CBR-based DSS provides similar, therefore representative, information. This is an important point, which must not be overlooked.

If it were possible to include prior probability information in the prognostic DSS, there is still the issue of understanding "probabilities". Several of the neonatologists commented on the need for large datasets, large sample sizes and more power, some even discussed problems associated with false positive and false negative results. These comments clearly indicate statistical knowledge and considerations. One must also keep in mind that the posterior 
probability is a probability conditional on variables specific to the individual case under consideration. For this reason, outcome probabilities from different cases do not necessarily apply to the case under consideration. However, if the models and the CBR can in fact present similar cases and the cases are representative of the patient for whom a query is made, then the DSS output will prove beneficial.

It should be possible for a prognostic DSS to rely on a large set of variables for each patient. Currently the Canadian Neonatal database contains over 200 different categories of information for each case. Not all of the categories have recorded values. The availability of NNs for estimating missing values will facilitate the development of a DSS that can bank on more variables. However, it too, carries a new set of possibilities for errors. While it is possible to increase the available information in a DSS output, it has been shown in the literature that more information does not necessarily lead to better decisions, and in some circumstances can detract from the accuracy of the clinician's judgement. The increase in the amount of information leads to an increase in confidence, but not necessarily in accuracy.

Also, if and when the required information is not available, the reconstructive nature of memory may prompt the neonatologists to fill in the gaps by making inferences if they use availability heuristic as a rule of thumb. A DSS's output can potentially bring about problems associated with availability heuristics. When a neonatologist views a DSS's output with data similar to her patient's data, she may overestimate the probability of an outcome based on information from the output.

Almost all interviewees believed that their confidence in their own judgment is not likely to be affected by the output of a DSS. However, they granted that if the DSS's output 
agreed with their hypothesis about a patient's outcome, they were more likely to have increased confidence in their own judgement. The reverse held true only occasionally, and only for those with fewer years of experience in the field. The confirmation bias, discussed in the literature review section, suggests that decision makers have a tendency to prefer information consistent with their own hypothesis, over inconsistent information. Alas, this form of cognitive bias is difficult to eliminate. Therefore, similar information provided by the prognostic DSS, if in agreement with the physicians' hypothesis, will likely support the confirmation bias of the neonatologists interviewed in this study. It should be noted that even without a prognostic DSS, physicians are likely to seek the advice of their colleagues or consultants in the field. This similarly carries the potential for the confirmation bias. Given the prevalence of this bias, little can be done other than warning the users against it.

Some interviewees expressed the need for isolating data for each hospital, ICU, city, province, region, and country as the needs of a patient case might dictate. The reason for this was given as "different grapes from different areas give different kinds of wine". Others believed that time-lines were important and that values from dataset containing several years old information may not be as applicable today due to changes in health care and patient management. The interviewees also expressed the need to have information about long-term outcomes such as the health status of the child four or more years after having left the NICU. One interviewee stated "A lot of what I do is not just based on what is happening today and tomorrow but what can happen in ten years and what parents want and can do with their babies." 
Almost all interviewees expressed the need for the DSS's output to be based on a very large database that has been tested for potential errors. Some of these requirements seem to be contradictory, such as the requirement for the information to be based on a database that is both large and centre specific; recent yet containing information about long-term outcomes.

This implies that a prognostic DSS needs to be programmed in such a way as to allow for various requirements. In theory, it is feasible to have prognostic DSS searching information based on different criteria. In practice, organizing such a system might prove challenging to say the least. The challenge need not be the programming aspect of the work but the logistics of its execution. Even if it were possible to provide neonatologists with an ideal system providing all relevant information according to any requirement, access and assimilation of large amounts of information from a DSS output cannot be ensured. Furthermore, neonatologists will continue to be subject to the same cognitive deficiencies as the lay population and as likely to commit errors due to heuristic biases.

Questions of logistics involve the resource requirements and the associated costs of DSS development that is suitable for all users. Every new category of information added carries a new rate of error, both in terms of human and machine error and in terms of potential for false positive and false negative test results. One neonatologist contended the clinical environments and patient management procedures vary "week by week". If this holds true for all NICUs, the gathering of enough information to build a large and reliable database for a DSS becomes an extremely challenging task. This problem will be exacerbated if the data must also be centre-specific. Other logistic questions involve the role of the persons responsible for DSS and database development. Specific roles need to be assigned when data 
accuracy is such an important issue. At present, it is not clear how the accuracy of the NICU database for the CBR-ANN hybrid DSS can be verified and ensured. These questions need to be considered before the use of a prognostic DSS can become feasible. These questions must be considered when costs and system management issues are being measured.

\section{LIMITATIONS}

A number of challenges limited the scope of this thesis and the ability to generalize some of its results. The small sample of interviewed neonatologists was clearly less than what is normally needed for drawing decisive conclusions and especially generalizing the findings. Furthermore, the neonatologists did not see or use the DSS they were interviewed about. Although they were familiar with the concept of DSSs and efforts were made to make sure that all knew about the nature of the DSS, it is possible that the interviewees imagined the DSS differently.

Also, assumptions were made about the accuracy of the databases used in this study. Given the source of the database and efforts already made to clean the data and remove errors, it was presumed to be accurate. No independent testing of this assumption was carried out.

The analyses carried out in this thesis were not theory driven. They were statistical in nature without clinical consideration. The interviews with neonatologists shed light on the clinical aspects of the variables considered and they were discussed at length, however, the analyses remain solely statistical. 


\section{CONCLUSION}

There are clearly many advantages to the use of a prognostic DSS in a NICU. There are also a number of challenges that have been discussed at length. The use of a CBR-based DSS is likely to eliminate some problems associated with representativeness heuristics but care should be exercised with regards to biases arising from availability heuristics and also the confirmation bias which might affect clinicians' prognostic decision making. More research is needed to determine the different functionalities of a DSS that will be useful to neonatologists. Although the current SNAPPE-II and MIRG models have variables that make for good starting points as predictive models more investigation is needed to determine a predictive model that has both statistical and clinical significance, based on which a prognostic DSS can be effective in NICUs. The models considered in this thesis can be used for a general neonatal population and database but will not to perform as well for a population of low birth-weight neonates. A different model would likely need to be considered for the low birth-weight patient category. In addition to statistical investigation, involving neonatologists as subject matter experts must be sought to facilitate the development of an effective prognostic DSS. Furthermore, this involvement will likely help in eliminating some of the clinicians' biases toward the use of DSS in clinical settings. 


\section{APPENDIX A. Interview Protocol \\ Participants' Information}

1. Years of experience in neonatology:

2. How do you rate yourself as a computer user?

Novice

Intermediate

Skilled

Expert

3. What are some of the software packages you use?

Examples: MS Word, SPSS, Microsoft Power Point

4. What are some of the purposes for which you use computers?

Please check all that apply

Email

Study

Search for information

News

Entertainment

Other: 


\section{Interview Questions}

1- Do you think a prognostic decision support system would be a valuable tool for you? Why?

2- If such a system were available, do you see yourself likely to use it? Why? Under what circumstances?

3- Would you be you willing to type information into the system to retrieve information such as patient cases that are similar to your patient? Why? Under what circumstances?

4- As an example, these variables could be included in the information you retrieve, can you think of additional variables that might further improve prognostic accuracy?

5- Please answer this question by using a scale of 1 to 100 , where 1 means I wouldn't trust it at all and 100 means I would trust it completely.

To what extent would you trust the information, given that source data comes from medical databases put together by hospital NICU's?. Why?

Trust -.---- (scale of 1 to 100)

Reason:

To what extent would you find the information reliable? Why?

Trust ----- (scale of 1 to 100 )

Reason:

To what extent would you find the information correct? Why?

Trust ----- (scale of 1 to 100$)$

Reason: 
7- If you were to use a prognostic Decision Support System, do you think information gained from finding similarity in cases would affect your confidence in your prognosis? How or why?

8- If the information gained from similar cases were to affect your confidence, for example, if the prediction given by the system disagreed with your view, would you see yourself likely to change your patient management plan after using the system? Why?

9- In order to retrieve examples of patients similar to yours from a prognostic Decision Support System, and if you could match your patient's information on any variable, which would be the three most important variables to you?

- Small for Gestational Age

- Apgar at 5 minutes

- Lowest $\mathrm{pO}_{2} / \mathrm{FiO}_{2}$ ratio

- Birth Weight

- Presence of Seizures

- Lowest Serum PH

- Lowest Blood Pressure

- Highest Blood Pressure

- Lowest Urine Output
- Highest $\mathrm{pCO}_{2}$

- Lowest Temperature

- Lowest Platelet Count

- Highest Sodium

- Lowest Glucose

- Highest Respiratory Rate (Breaths/Min)

- Other:

- Other:

- Other:

10- If you were to use a prognostic decision-aid, what information would you like to be able to obtain (what findings would you want to have information on? 


\section{Sorting Variables in terms of their importance}

1- From the list below, please choose the three most important and the three least important variables for making a prognosis:

\section{- $\mathrm{PO} 2 \mathrm{FiO} 2 \mathrm{R}$}

- Lowest Urine Output

- Lowest blood pressure

- Seizure

- Lowest Serum

- $\quad$ APGAR at 5

- Lowest Platelet

- Small for Gestational Age

- Highest Sodium

- Highest Respiratory Rate

- Highest $\mathrm{PCO}_{2}$

- Birth Weight

- Lowest Glucose

- Lowest temperature

- Highest Blood Pressure 


\section{Department of Psychology: Carleton University}

\section{Informed Consent Form: The human role in decision support}

The purpose of an informed consent is to insure that you understand the purpose of the study and the nature of your involvement. The informed consent must provide sufficient information such that you have the opportunity to determine whether you wish to participate in the study.

\section{Present study: The human role in decision support}

\section{Purpose:}

The purpose of this interview is to investigate physicians' perception of technology, their potential acceptance and their predicted use of a prognostic decision support system. Results of this interview will be used in a research project.

\section{Task requirements:}

I will ask your opinions about your use of technology and perception of prognostic decision support systems. I will ask you for your reasons for your responses. The interview session will be tape recorded and later transcribed ad verbatim.

I will ask you to sort out a set of variables in terms of their importance for making prognostic decisions for neonatal patients admitted to a Neonatal Intensive Care Unit.

I will ask you for three case examples of hypothetical patients and the values you would assign a set of variables for these hypothetical patients.

You will also be asked to complete a short demographic questionnaire.

All of your responses will be recorded anonymously.

\section{Duration and locale:}

The interview session will last approximately 40 minutes. Testing will take place in a location convenient to interviewees at their place of work.

\section{Potential risk/discomfort:}

There are no potential physical or psychological risks in these interviews.

\section{Anonymity/confidentiality:}

The data collected in these interviews are confidential. All data are coded such that your name is not associated with the data. In addition, the coded data are made available only to the researchers associated with this project. They will not be shared with supervising staff at the hospital. In the event that these results are published confidentiality and anonymity of participants will be maintained, and only aggregate results will be reported. Your decision to participate or not in this interview will be kept confidential.

\section{Right to withdraw:}

Your participation is entirely voluntary. You have the right to withdraw from the experiment at any time without penalty. 


\section{Signatures}

I have read the above description of "The human role in decision support" study and understand the conditions of my participation. My signature indicates that I agree to participate in this experiment and $\mathrm{I}$ agree to allow the interviewer to audiotape this session.

Participant's Name (please print):

Participant's Signature:

Date:

Researcher's name and signature: 


\section{Debriefing}

The purpose of this study is to examine the feasibility of use of a prognostic decision support system in a Neonatal Intensive Care Unit environment and system reception by experts.

Many decision support systems are currently available to the medical community some of which are in use while others are being developed and investigated as potentially useful tools that could enhance medical decision making. Research suggests that the majority of these systems have been tested from a system and not a human perspective. This work attempts to study experts' perceptions, attitudes, and potential use of decision support systems.

We hope our results will shed some light on these questions about what factors experts take into account when faced with the decision to use and incorporate a prognostic decision support system into their practice.

We would like to thank you for participating in this research. Your time and effort are greatly appreciated!

If you have any question or comments about this research, then please feel free to contact $x x x x$ Should you have any ethical or other concerns about this study then please contact xxx Chair, Carleton University Research Ethics Committee for Psychological Research, or xxx (Chair, Department of Psychology). 
APPENDIX B. Full Correlation Matrix

\begin{tabular}{|c|c|c|c|c|c|c|c|c|c|c|c|c|c|c|}
\hline \multicolumn{15}{|c|}{ Correlations } \\
\hline & & $\begin{array}{l}\text { LBLO } \\
\text { ODP }\end{array}$ & $\begin{array}{c}\text { LTEM } \\
\text { PF }\end{array}$ & $\begin{array}{c}\text { PO2FI } \\
\text { O2R }\end{array}$ & $\begin{array}{l}\text { LURI } \\
\text { NE }\end{array}$ & $\begin{array}{l}\text { LSER } \\
\text { UM }\end{array}$ & $\begin{array}{c}\text { SEIZU } \\
\text { RE }\end{array}$ & $\begin{array}{c}\text { BTHW } \\
T\end{array}$ & $\begin{array}{c}\text { APGA } \\
\text { R5 }\end{array}$ & SGA & MORT & $\begin{array}{c}\text { HPCO } \\
2\end{array}$ & $\begin{array}{l}\text { LGLU } \\
\qquad \mathrm{C}\end{array}$ & $\begin{array}{l}\text { HBLO } \\
\text { ODP }\end{array}$ \\
\hline $\begin{array}{l}\mathrm{L} \\
\mathrm{B}\end{array}$ & $\begin{array}{l}\text { Pearson } \\
\text { Correlation }\end{array}$ & 1.000 & $.368 \dagger$ & $.155 \dagger$ & $.103 \dagger$ & $.190 \dagger$ & $.070 \dagger$ & $.644 \dagger$ & $.137 \dagger$ & $-.078 t$ & $.156 \dagger$ & $.096 t$ & $.101 t$ & $.614 \dagger$ \\
\hline 0 & Sig. (2-tailed) & & .000 & .000 & .000 & .000 & .001 & .000 & .000 & .000 & .000 & .000 & .000 & .000 \\
\hline $\mathbf{P}$ & N & 2402 & 2402 & 2402 & 2402 & 2402 & 2402 & 2402 & 2402 & 2402 & 2402 & 2402 & 2402 & 2402 \\
\hline $\begin{array}{l}\mathrm{L} \\
\mathrm{T}\end{array}$ & $\begin{array}{l}\text { Pearson } \\
\text { Correlation }\end{array}$ & $.368 \dagger$ & 1.000 & $.056 \dagger$ & $.106 \dagger$ & $.142 \dagger$ & .025 & $.441 \dagger$ & $.140 \dagger$ & $-.088 \dagger$ & $.219 \dagger$ & -.040 & .006 & $.250 \dagger$ \\
\hline M & Sig. (2-tailed) & .000 & & .006 & .000 & .000 & .215 & .000 & .000 & .000 & .000 & .052 & .778 & .000 \\
\hline $\mathbf{F}$ & $N$ & 2402 & 2402 & 2402 & 2402 & 2402 & 2402 & 2402 & 2402 & 2402 & 2402 & 2402 & 2402 & 2402 \\
\hline $\begin{array}{l}P \\
0\end{array}$ & $\begin{array}{l}\text { Pearson } \\
\text { Correlation }\end{array}$ & $.155 \dagger$ & $.056 \dagger$ & 1.000 & .038 & $.326 \dagger$ & $.103 \dagger$ & .032 & $.115 \dagger$ & $.049^{*}$ & $.140 \dagger$ & $-295 \dagger$ & .006 & -.039 \\
\hline $\mathbf{F}$ & Sig. (2-tailed) & .000 & .006 & . & .059 & .000 & .000 & .122 & .000 & .017 & .000 & .000 & .778 & .053 \\
\hline 2 & N & 2402 & 2402 & 2402 & 2402 & 2402 & 2402 & 2402 & 2402 & 2402 & 2402 & 2402 & 2402 & 2402 \\
\hline $\mathbf{L}$ & $\begin{array}{l}\text { Pearson } \\
\text { Correlation }\end{array}$ & $.103 \dagger$ & $.106 \dagger$ & .038 & 1.000 & $.101 \dagger$ & $-.074 \dagger$ & $.051^{*}$ & $.128 \dagger$ & .029 & $.121 \dagger$ &. .027 & $.061 \dagger$ & $.062 \dagger$ \\
\hline
\end{tabular}




\begin{tabular}{|c|c|c|c|c|c|c|c|c|c|c|c|c|c|c|}
\hline I & Sig. (2-tailed) & .000 & .000 & .059 & & .000 & .000 & .013 & .000 & .150 & .000 & .182 & .003 & .002 \\
\hline E & $\mathbf{N}$ & 2402 & 2402 & 2402 & 2402 & 2402 & 2402 & 2402 & 2402 & 2402 & 2402 & 2402 & 2402 & 2402 \\
\hline $\begin{array}{l}\text { L } \\
\text { S }\end{array}$ & $\begin{array}{l}\text { Pearson } \\
\text { Correlation }\end{array}$ & $.190 \dagger$ & $.142 \dagger$ & $.326 \dagger$ & $.101 \dagger$ & 1.000 & .004 & $.138 \dagger$ & $.236 \dagger$ & -.012 & $.242 \dagger$ & $.706 \dagger$ & .009 & $.075 \dagger$ \\
\hline $\mathbf{R}$ & Sig. (2-tailed) & .000 & .000 & .000 & .000 & & .838 & .000 & .000 & .543 & .000 & .000 & .645 & .000 \\
\hline$M$ & N & 2402 & 2402 & 2402 & 2402 & 2402 & 2402 & 2402 & 2402 & 2402 & 2402 & 2402 & 2402 & 2402 \\
\hline $\begin{array}{l}S \\
E\end{array}$ & $\begin{array}{l}\text { Pearson } \\
\text { Correlation }\end{array}$ & $.070 \dagger$ & .025 & $.103 \dagger$ & $-.074 \dagger$ & .004 & 1.000 & $.157 \dagger$ & $-.194 \dagger$ & .014 & $-.084 \dagger$ & $-095 \dagger$ & -.011 & $.092 \dagger$ \\
\hline $\mathbf{z}$ & Sig. (2-tailed) & .001 & .215 & .000 & .000 & .838 & & .000 & .000 & .489 & .000 & .000 & .582 & .000 \\
\hline E & $\mathbf{N}$ & 2402 & 2402 & 2402 & 2402 & 2402 & 2402 & 2402 & 2402 & 2402 & 2402 & 2402 & 2402 & 2402 \\
\hline B & $\begin{array}{l}\text { Pearson } \\
\text { Correlation }\end{array}$ & $.644 \dagger$ & .441† & .032 & $-.051^{*}$ & $.138 \dagger$ & $.157 \dagger$ & 1.000 & $.049^{*}$ & $-.182 \dagger$ & $.136 \dagger$ & $-.084 \dagger$ & $.065 \dagger$ & $.570 \dagger$ \\
\hline H & Sig. (2-tailed) & .000 & .000 & .122 & .013 & .000 & .000 & & .015 & .000 & .000 & .000 & .002 & .000 \\
\hline & $\mathbf{N}$ & 2402 & 2402 & 2402 & 2402 & 2402 & 2402 & 2402 & 2402 & 2402 & 2402 & 2402 & 2402 & 2402 \\
\hline $\begin{array}{l}\text { A } \\
\text { P }\end{array}$ & $\begin{array}{l}\text { Pearson } \\
\text { Correlation }\end{array}$ & $.137 \dagger$ & $.140 \dagger$ & $.115 t$ & $.128 \dagger$ & $.236 \dagger$ & - $194 \dagger$ & $.049^{*}$ & 1.000 & .022 & $.258 \dagger$ & $-.051^{*}$ & .006 & .014 \\
\hline A & Sig. (2-tailed) & .000 & .000 & .000 & .000 & .000 & .000 & .015 & . & .271 & .000 & .013 & .786 & .494 \\
\hline
\end{tabular}




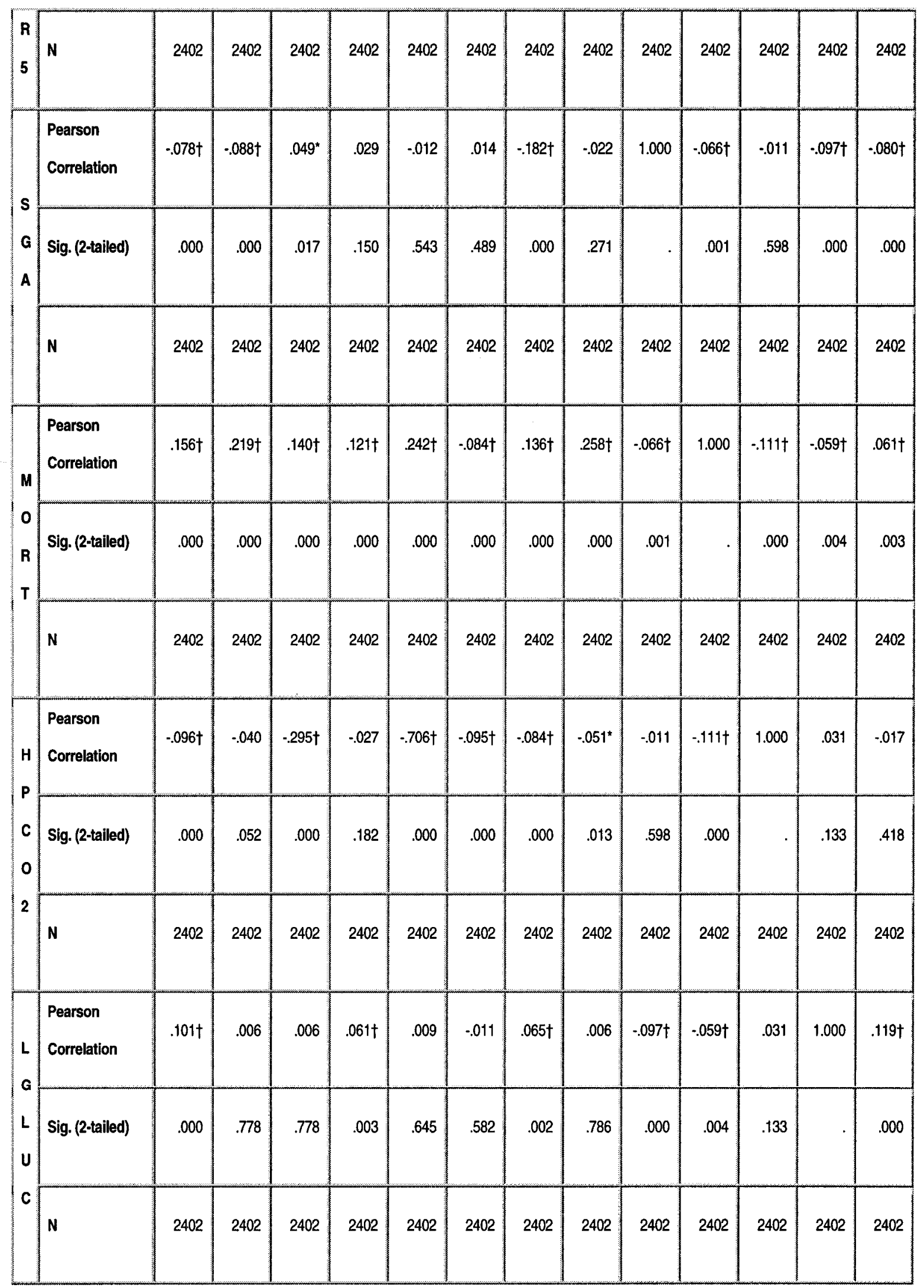




\begin{tabular}{|c|c|c|c|c|c|c|c|c|c|c|c|c|c|c|}
\hline H & $\begin{array}{l}\text { Pearson } \\
\text { Correlation }\end{array}$ & $.614 \dagger$ & $.250 \dagger$ & -.039 & $.062 \dagger$ & $.075 t$ & $.092 \dagger$ & $.570 \dagger$ & .014 & $-.080 \dagger$ & $.061 \dagger$ & -.017 & $.119 \dagger$ & 1.000 \\
\hline 0 & Sig. (2-tailed) & .000 & .000 & .053 & .002 & .000 & .000 & .000 & .494 & .000 & .003 & .418 & .000 & . \\
\hline $\mathbf{p}$ & $\mathbf{N}$ & 2402 & 2402 & 2402 & 2402 & 2402 & 2402 & 2402 & 2402 & 2402 & 2402 & 2402 & 2402 & 2402 \\
\hline \multicolumn{15}{|c|}{ " Correlation is significant at the 0.01 level (2-tailed). } \\
\hline & lation is sign & it the & EVe & ailed). & & & & & & & & & & \\
\hline
\end{tabular}


APPENDIX C. List of Interaction Terms

\begin{tabular}{|l|l|}
\hline Variable ID & Variable description \\
\hline V1 & Zscore(LBLOODP) \\
\hline V2 & Zscore(LTEMPF) \\
\hline V3 & Zscore(PO2FIO2R) \\
\hline V4 & Zscore(LURINE) \\
\hline V5 & Zscore(LSERUM) \\
\hline V6 & Zscore(SEIZURE) \\
\hline V7 & Zscore(BTHWT) \\
\hline V8 & Zscore(APGAR5) \\
\hline V9 & Zscore(SGA) \\
\hline V10 & Zscore(LPLT) \\
\hline V11 & Zscore(HSODIUM) \\
\hline V12 & Zscore(HRESPR) \\
\hline V13 & Zscore(HPCO2) \\
\hline V14 & Zscore(LGLUC) \\
\hline V15 & Zscore(HBLOODP) \\
\hline & \\
\hline
\end{tabular}


Interaction Table for the SNAPPE-II model

\begin{tabular}{|c|c|c|c|c|c|c|c|c|c|}
\hline \multicolumn{10}{|c|}{ Variables in the Equation } \\
\hline & & \multirow[t]{2}{*}{ B } & \multirow{2}{*}{ S.E. } & \multirow[t]{2}{*}{ Wald } & \multirow[t]{2}{*}{ df } & \multirow{2}{*}{ Sig. } & \multirow{2}{*}{$\operatorname{Exp}(\mathbf{B})$} & \multicolumn{2}{|c|}{$\begin{array}{l}\text { 95.0\% C.I.for } \\
\text { EXP(B) }\end{array}$} \\
\hline & & & & & & & & Lower & Upper \\
\hline \multirow{22}{*}{$\begin{array}{l}\text { Step } \\
\text { 1(a) }\end{array}$} & v1 & .158 & .142 & 1.235 & 1 & .266 & 1.171 & .886 & 1.548 \\
\hline & v2 & .349 & .128 & 7.400 & 1 & .007 & 1.417 & 1.102 & 1.822 \\
\hline & v3 & .453 & .143 & 10.081 & 1 & .001 & 1.573 & 1.189 & 2.080 \\
\hline & v4 & .539 & .106 & 25.701 & 1 & .000 & 1.715 & 1.392 & 2.112 \\
\hline & v5 & 1.089 & 494 & 4.860 & 1 & .027 & 2.971 & 1.128 & 7.821 \\
\hline & v6 & .345 & .162 & 4.554 & 1 & .033 & 1.412 & 1.029 & 1.938 \\
\hline & v7 & .555 & .088 & 39.431 & 1 & .000 & 1.741 & 1.465 & 2.070 \\
\hline & v8 & .229 & .736 & .096 & 1 & .756 & 1.257 & .297 & 5.318 \\
\hline & V1V2 & .158 & .112 & 1.999 & 1 & & 1.171 & .941 & 1.457 \\
\hline & V1V3 & .349 & .126 & 6.226 & 1 & .013 & .730 & .570 & .935 \\
\hline & V1V4 & .453 & .135 & 192 & 1 & & 1.061 & .814 & 1.383 \\
\hline & V1V5 & .539 & .097 & .271 & 1 & & 1.052 & .869 & 1.274 \\
\hline & V1V6 & 1.089 & .081 & 1.290 & 1 & & 1.096 & .936 & 1.283 \\
\hline & V1V7 & .345 & .101 & 5.897 & 1 & .015 & .783 & .643 & .954 \\
\hline & V1V8 & .555 & .093 & 4.767 & 1 & .029 & 1.226 & 1.021 & 1.471 \\
\hline & V1V9 & .229 & .087 & .731 & 1 & & .928 & .783 & 1.101 \\
\hline & V2V3 & .158 & .104 & 1.274 & 1 & & .889 & .724 & 1.091 \\
\hline & V2V4 & -.113 & .107 & 1.112 & 1 & & .894 & .725 & 1.102 \\
\hline & V2V5 & .001 & .064 & .000 & 1 & & 1.001 & .883 & 1.135 \\
\hline & V2V6 & .051 & .064 & .617 & 1 & & 1.052 & .927 & 1.193 \\
\hline & V2V7 & -.213 & .109 & 3.828 & 1 & .050 & .808 & .652 & 1.000 \\
\hline & V2V8 & .077 & .065 & 1.389 & 1 & & 1.080 & .950 & 1.226 \\
\hline
\end{tabular}




\begin{tabular}{|c|c|c|c|c|c|c|c|c|c|}
\hline & V2V9 & -.189 & .073 & 6.613 & 1 & .010 & .828 & .717 & .956 \\
\hline & V3V4 & .214 & .154 & 1.938 & 1 & & 1.239 & .916 & 1.675 \\
\hline & V3V5 & -.091 & .085 & 1.166 & 1 & & .913 & .773 & 1.077 \\
\hline & V3V6 & -.058 & .066 & .763 & 1 & & .944 & .829 & 1.075 \\
\hline & V3V7 & .218 & .160 & 1.861 & 1 & & 1.244 & .909 & 1.702 \\
\hline & V3V8 & -.119 & .088 & 1.821 & 1 & & .888 & .747 & 1.055 \\
\hline & V3V9 & .214 & .110 & 3.785 & 1 & & 1.238 & .998 & 1.535 \\
\hline & V4V5 & .036 & .092 & .156 & 1 & & 1.037 & .866 & 1.242 \\
\hline & V4V6 & -.116 & .099 & 1.384 & 1 & & .890 & .733 & 1.081 \\
\hline & V4V7 & .092 & .149 & .376 & 1 & & 1.096 & .818 & 1.469 \\
\hline & V4V8 & -.005 & .096 & .003 & 1 & & .995 & .824 & 1.201 \\
\hline & V4V9 & .189 & .112 & 2.861 & 1 & & 1.209 & .970 & 1.505 \\
\hline & V5V6 & -.092 & .051 & 3.325 & 1 & & .912 & .826 & 1.007 \\
\hline & V5V7 & .008 & .111 & .005 & 1 & & 1.008 & .810 & 1.253 \\
\hline & V5V8 & .142 & .049 & 8.414 & 1 & .004 & 1.153 & 1.047 & 1.269 \\
\hline & V5V9 & -.109 & .069 & 2.471 & 1 & & .897 & .783 & 1.027 \\
\hline & V6V7 & -.011 & .087 & .016 & 1 & & .989 & .834 & 1.173 \\
\hline & V6V8 & .023 & .051 & .214 & 1 & & 1.024 & .927 & 1.130 \\
\hline & V6V9 & .056 & .055 & 1.048 & 1 & & 1.057 & .950 & 1.177 \\
\hline & V7V8 & -.073 & .106 & .468 & 1 & & .930 & .755 & 1.145 \\
\hline & V7V9 & .119 & .137 & .754 & 1 & & 1.126 & .862 & 1.471 \\
\hline & V8V9 & .103 & .077 & 1.780 & 1 & & 1.109 & .953 & 1.290 \\
\hline & Constant & 1.819 & .853 & 4.550 & 1 & .033 & 6.168 & & \\
\hline \multicolumn{10}{|c|}{$\begin{array}{l}\text { a Variable(s) entered on step 1: V1V2, V1V3, V1V4, V1V5, V1V6, V1V7, V1V8, } \\
\text { V1V9, V2V3, V2V4, V2V5, V2V6, V2V7, V2V8, V2V9, V3V4, V3V5, V3V6, V3V7, } \\
\text { V3V8, V3V9, V4V5, V4V6, V4V7, V4V8, V4V9, V5V6, V5V7, V5V8, V5V9, V6V7, } \\
\text { V6V8, V6V9, V7V8, V7V9, V8V9. }\end{array}$} \\
\hline
\end{tabular}


Interaction Table for the MIRG model

\begin{tabular}{|c|c|c|c|c|c|c|c|c|c|}
\hline & & \multirow[t]{2}{*}{ B } & \multirow[t]{2}{*}{ S.E. } & \multirow[t]{2}{*}{ Wald } & \multirow[t]{2}{*}{ df } & \multirow[t]{2}{*}{ Sig. } & \multirow[t]{2}{*}{$\operatorname{Exp}(\mathbf{B})$} & \multicolumn{2}{|c|}{$\begin{array}{l}\text { 95.0\% C.I.for } \\
\text { EXP(B) }\end{array}$} \\
\hline & & & & & & & & Lower & Upper \\
\hline \multirow{22}{*}{$\begin{array}{l}\text { Step } \\
\text { 1(a) }\end{array}$} & V2 & .061 & . 144 & .182 & 1 & .669 & 1.063 & .802 & 1.410 \\
\hline & v3 & .170 & .144 & 1.404 & 1 & .236 & 1.186 & .895 & 1.571 \\
\hline & V4 & .726 & .179 & 16.525 & 1 & .000 & 2.067 & 1.457 & 2.934 \\
\hline & V5 & .221 & .192 & 1.329 & 1 & .249 & 1.248 & .857 & 1.817 \\
\hline & V7 & .360 & .173 & 4.348 & 1 & .037 & 1.433 & 1.022 & 2.010 \\
\hline & V8 & .498 & .109 & 20.815 & 1 & .000 & 1.646 & 1.329 & 2.039 \\
\hline & V9(1) & -.288 & .892 & .104 & 1 & .747 & .749 & .130 & 4.309 \\
\hline & V10 & .372 & .122 & 9.323 & 1 & .002 & 1.451 & 1.142 & 1.842 \\
\hline & V11 & -.396 & .119 & 11.109 & 1 & .001 & .673 & .533 & .849 \\
\hline & V12 & .503 & .133 & 14.234 & 1 & .000 & 1.653 & 1.273 & 2.147 \\
\hline & V13 & .180 & .194 & .868 & 1 & .352 & 1.198 & .819 & 1.750 \\
\hline & V14 & -.187 & .107 & 3.039 & 1 & .081 & .829 & .672 & 1.024 \\
\hline & V15 & .013 & . 141 & .009 & 1 & .926 & 1.013 & .768 & 1.336 \\
\hline & V2V3 & -.136 & .110 & 1.520 & 1 & .218 & .873 & .703 & 1.084 \\
\hline & V2V4 & -.102 & .123 & .691 & 1 & .406 & .903 & .709 & 1.149 \\
\hline & V2V5 & -.049 & .111 & .194 & 1 & .660 & .952 & .766 & 1.184 \\
\hline & V2V7 & -.107 & . 108 & .992 & 1 & .319 & .898 & .728 & 1.109 \\
\hline & V2V8 & -.028 & .071 & .157 & 1 & .692 & .972 & .846 & 1.117 \\
\hline & V2V9 & -.075 & .082 & .841 & 1 & .359 & .928 & .790 & 1.089 \\
\hline & V3V4 & .268 & .181 & 2.196 & 1 & .138 & 1.308 & .917 & 1.865 \\
\hline & V3V5 & .188 & .147 & 1.638 & 1 & .201 & 1.207 & .905 & 1.610 \\
\hline & V3V7 & -.028 & .166 & .028 & 1 & .868 & .973 & .702 & 1.347 \\
\hline
\end{tabular}




\begin{tabular}{|c|c|c|c|c|c|c|c|c|}
\hline V3V8 & -.140 & .106 & 1.752 & 1 & .186 & .869 & .706 & 1.070 \\
\hline V3V9 & .116 & .117 & .989 & 1 & .320 & 1.123 & .893 & 1.412 \\
\hline V4V5 & -.094 & .167 & .318 & 1 & .573 & .910 & .657 & 1.262 \\
\hline V4V7 & -.141 & .173 & .662 & 1 & .416 & .869 & .619 & 1.219 \\
\hline V4V8 & .124 & .109 & 1.305 & 1 & .253 & 1.132 & .915 & 1.401 \\
\hline V4V9 & .065 & .123 & .282 & 1 & .596 & 1.067 & .839 & 1.357 \\
\hline V5V7 & .092 & .152 & .366 & 1 & .545 & 1.097 & .814 & 1.478 \\
\hline V5V8 & .009 & .082 & .011 & 1 & .915 & 1.009 & .858 & 1.186 \\
\hline V5V9 & -.029 & .112 & .065 & 1 & .798 & .972 & .781 & 1.209 \\
\hline V7V8 & .016 & .105 & .024 & 1 & .878 & 1.016 & .827 & 1.249 \\
\hline V7V9 & .144 & .171 & .712 & 1 & .399 & 1.155 & .826 & 1.615 \\
\hline V8V9 & .113 & .085 & 1.774 & 1 & .183 & 1.120 & .948 & 1.323 \\
\hline V10V11 & .165 & .100 & 2.720 & 1 & .099 & 1.179 & .969 & 1.435 \\
\hline V10V12 & .032 & .115 & .079 & 1 & .779 & 1.033 & .825 & 1.293 \\
\hline V10V13 & -.057 & .112 & .261 & 1 & .609 & .944 & .758 & 1.176 \\
\hline V10V14 & -.254 & .085 & 8.857 & 1 & .003 & .776 & .656 & .917 \\
\hline V10V15 & -.154 & .113 & 1.849 & 1 & .174 & .858 & .687 & 1.070 \\
\hline V11V12 & -.141 & .108 & 1.699 & 1 & .192 & .869 & .703 & 1.073 \\
\hline V11V13 & .122 & .113 & 1.168 & 1 & .280 & 1.129 & .906 & 1.408 \\
\hline V11V14 & -.027 & .059 & .203 & 1 & .653 & .974 & .867 & 1.094 \\
\hline V11V15 & .040 & .104 & .145 & 1 & .703 & 1.041 & .848 & 1.276 \\
\hline V12V13 & -.119 & .140 & .726 & 1 & .394 & .887 & .674 & 1.168 \\
\hline V12V14 & -.124 & .093 & 1.789 & 1 & .181 & .883 & .736 & 1.060 \\
\hline V12V15 & -.411 & .131 & 9.852 & 1 & .002 & .663 & .513 & .857 \\
\hline V13V14 & .064 & .086 & .546 & 1 & .460 & 1.066 & .900 & 1.262 \\
\hline V13V15 & -.106 & .121 & .773 & 1 & .379 & .899 & .710 & 1.139 \\
\hline V14V15 & -.106 & .092 & 1.338 & 1 & .247 & .899 & .751 & 1.076 \\
\hline
\end{tabular}




\begin{tabular}{|c|c|c|c|c|c|c|c|c|}
\hline V2V10 & -.062 & .105 & .345 & 1 & .557 & .940 & .766 & 1.154 \\
\hline V2V11 & .044 & .072 & .372 & 1 & .542 & 1.045 & .907 & 1.204 \\
\hline V2V12 & .244 & .116 & 4.432 & 1 & .035 & 1.276 & 1.017 & 1.602 \\
\hline V2V13 & -.177 & .108 & 2.660 & 1 & .103 & .838 & .678 & 1.036 \\
\hline V2V14 & .223 & .084 & 7.045 & 1 & .008 & 1.250 & 1.060 & 1.475 \\
\hline V2V15 & -.063 & .107 & .348 & 1 & .555 & .939 & .762 & 1.157 \\
\hline V3V10 & -.186 & .115 & 2.613 & 1 & .106 & .830 & .662 & 1.040 \\
\hline V3V11 & .117 & .114 & 1.058 & 1 & .304 & 1.124 & .899 & 1.406 \\
\hline V3V12 & .136 & .131 & 1.081 & 1 & .298 & 1.145 & .887 & 1.480 \\
\hline V3V13 & .169 & .146 & 1.333 & 1 & .248 & 1.184 & .889 & 1.578 \\
\hline V3V14 & .081 & .100 & .658 & 1 & .417 & 1.084 & .892 & 1.319 \\
\hline V3V15 & -.029 & .143 & .042 & 1 & .837 & .971 & .733 & 1.286 \\
\hline V4V10 & .116 & .128 & .818 & 1 & .366 & 1.123 & .874 & 1.443 \\
\hline V4V11 & .072 & .093 & .596 & 1 & .440 & 1.075 & .895 & 1.291 \\
\hline V4V12 & -.038 & .150 & .066 & 1 & .797 & .962 & .717 & 1.291 \\
\hline V4V13 & -.002 & .153 & .000 & 1 & .988 & .998 & .740 & 1.346 \\
\hline V4V14 & -.216 & .097 & 4.889 & 1 & .027 & .806 & .666 & .976 \\
\hline V4V15 & .330 & .157 & 4.417 & 1 & .036 & 1.391 & 1.022 & 1.892 \\
\hline V5V10 & -.212 & .118 & 3.247 & 1 & .072 & .809 & .642 & 1.019 \\
\hline V5V11 & .138 & .118 & 1.370 & 1 & .242 & 1.148 & .911 & 1.446 \\
\hline V5V12 & -.163 & .157 & 1.084 & 1 & .298 & .849 & .625 & 1.155 \\
\hline V5V13 & .100 & .045 & 5.028 & 1 & .025 & 1.105 & 1.013 & 1.206 \\
\hline V5V14 & .126 & .098 & 1.641 & 1 & .200 & 1.134 & .935 & 1.376 \\
\hline V5V15 & -.004 & .126 & .001 & 1 & .972 & .996 & .778 & 1.275 \\
\hline V7V10 & .120 & .137 & .774 & 1 & .379 & 1.128 & .863 & 1.474 \\
\hline V7V11 & .021 & .111 & .035 & 1 & .852 & 1.021 & .821 & 1.270 \\
\hline V7V12 & -.080 & .146 & .305 & 1 & .581 & .923 & .694 & 1.228 \\
\hline
\end{tabular}




\begin{tabular}{|c|c|c|c|c|c|c|c|c|c|}
\hline & V7V13 & .286 & .158 & 3.295 & 1 & .069 & 1.332 & .977 & 1.814 \\
\hline & V7V14 & -.018 & .112 & .027 & 1 & .870 & .982 & .789 & 1.222 \\
\hline & V7V15 & -.007 & .109 & .005 & 1 & .945 & .993 & .801 & 1.229 \\
\hline & V8V10 & .117 & .090 & 1.694 & 1 & .193 & 1.124 & .943 & 1.339 \\
\hline & V8V11 & -.186 & .075 & 6.209 & 1 & .013 & .831 & .718 & .961 \\
\hline & V8V12 & -.120 & .099 & 1.489 & 1 & .222 & .887 & .731 & 1.076 \\
\hline & V8V13 & -.178 & .081 & 4.802 & 1 & .028 & .837 & .714 & .981 \\
\hline & V8V14 & .086 & .067 & 1.632 & 1 & .201 & 1.089 & .955 & 1.242 \\
\hline & V8V15 & .262 & .099 & 6.987 & 1 & .008 & 1.299 & 1.070 & 1.577 \\
\hline & V9V10 & -.002 & .089 & .000 & 1 & .983 & .998 & .838 & 1.189 \\
\hline & V9V11 & .093 & .071 & 1.721 & 1 & .190 & 1.097 & .955 & 1.261 \\
\hline & V9V12 & .050 & .116 & .184 & 1 & .668 & 1.051 & .838 & 1.319 \\
\hline & V9V13 & .135 & .114 & 1.404 & 1 & .236 & 1.145 & .915 & 1.431 \\
\hline & V9V14 & .046 & .101 & .206 & 1 & .650 & 1.047 & .858 & 1.278 \\
\hline & V9V15 & -.143 & .089 & 2.610 & 1 & .106 & .867 & .728 & 1.031 \\
\hline & Constant & 3.625 & .895 & 16.398 & 1 & .000 & 37.541 & & \\
\hline $\begin{array}{l}\text { a Varia } \\
\text { V3V5, } \\
\text { V7V9, } \\
\text { V11V1 } \\
\text { V2V11 } \\
\text { V3V15 } \\
\text { V5V13 } \\
\text { V8V11 } \\
\text { V9V15 }\end{array}$ & $\begin{array}{l}\text { ble(s) entere } \\
\text { V3V7, V3V } \\
\text { V8V9, V10 } \\
4, \mathrm{~V} 11 \mathrm{~V} 15, \\
\text {, V2V12, V' } \\
\text {, V4V10, V } \\
\text {, V5V14, V } \\
\text {, V8V12, V } \\
\text {. }\end{array}$ & $\begin{array}{l}\text { on step } \\
\text { V3V9, } \\
1, \text { V10 } \\
12 \text { V13, } \\
13, \text { V2 } \\
11, \text { V4 } \\
15, \text { V7 } \\
13, \text { V8 }\end{array}$ & $\begin{array}{l}12, \mathrm{~V} \\
\mathrm{~V} 12 \mathrm{~V} \\
14, \mathrm{~V} \\
12, \mathrm{~V} \\
10, \mathrm{~V} \\
14, \mathrm{~V}\end{array}$ & $\begin{array}{l}3, \mathrm{~V} 2 \mathrm{~V} 4, \\
\mathrm{~V} 4 \mathrm{~V} 7, \mathrm{~V} 4 \\
0 \mathrm{~V} 13, \mathrm{~V} 1 \\
4, \mathrm{~V} 12 \mathrm{~V} 1 \\
\mathrm{~V} 15, \mathrm{~V} 3 \\
\mathrm{~V} 13, \mathrm{~V} 4 \\
\mathrm{~V} 11, \mathrm{~V} 7 \\
\mathrm{~V} 15, \mathrm{~V} 9\end{array}$ & & $\begin{array}{l}\text { 13V14 } \\
\text { V3V1 } \\
\text { V4V1 } \\
\text { V7V1 } \\
\text { V9V1 }\end{array}$ & $\begin{array}{l}15, \mathrm{~V} 11 \\
\text { V13V15, } \\
\text { V3V12, } \\
\text { V5V10, } \\
\text { V7V14, } \\
\text { V9V12, }\end{array}$ & $\begin{array}{l}4, \mathrm{~V} 11 \mathrm{~V} \\
4 \mathrm{~V} 15, \\
\mathrm{~V} 13, \mathrm{~V} \\
\mathrm{~V} 11, \mathrm{~V} \\
\mathrm{~V} 15, \mathrm{~V} \\
\mathrm{~V} 13, \mathrm{~V}\end{array}$ & $\begin{array}{l}7 \mathrm{~V} 8, \\
10, \\
14, \\
12, \\
10, \\
14,\end{array}$ \\
\hline
\end{tabular}


Reference List

The Canadian NICU Network (2003). Website [On-line]. Available:

http://www.bcricwh.bc.ca/CES/snap.html

Arts, d. G., Bosman, R. J., Jonge, E., \& Keizer, N. (2003). Training in data definitions improves quality of intensive care data. Critical Care, 7.

Bell, D. E., Raiffa, H., \& Tversky, A. (1988). Decision making

descriptive, normative, and prescriptive interactions. Cambridge: Cambridge University Press.

Beyth-Marom \& Fischhoff, B. (1983). Diagnosticity and pseudodiagnosticity. Journal of Personality and Social Psychology, 45, 1185-1195.

Boon, M. E. \& Kok, L. P. (1995). Histological validation of neural-network assisted cervical screening: a comparison with the conventional approach. Cell Vision, 2, 23-27.

Christensen-Szalanski, J. J. J., Beck, D. E., Christensen-Szalanski, C. M., \& Koepsell, T. D. (1983). The effect of journal coverage on physicians' perception of risk. Journal of Applied Psychology, 68, 278-284.

Civetta, J. M. (1997). Prediction and Definition of outcome. In J.M.Civetta (Ed.), Critical Care (3 ed., pp. 1873-1898). Lippincott Williams \& Wilkins. 
Dawes, R. M. (1971). A case study of graduate admissions: Application of three principles of human decision making. American Psychologist, 26, 180-188.

Dawes, R. M. (2001). The robust beauty of improper linear models in decision making" in Kahneman, D, Slovic, P., Tversky, A. (eds.) Judgment under uncertainty: Heuristics and biases. Cambridge University Press. In D.Kahneman \& A. Tversky (Eds.), (pp. 391-407).

Dawes, R. M. \& Kagan, J. (1988). Rational choice in an uncertain world. San Diego: Harcourt Brace Jovanovich.

De Vaul, R., Jervey F., Chappell, J., Carver, P., Short, B., \& O'Keefe, S. (1957). Medical school performance of initially rejected students. Journal of the American Medical Association, 257, 47-51.

Ebert, R. J. \& Kruse, T. E. (1978). Bootstrapping the Security Analyst. Journal of Applied Psychology., 63, 117.

Eddy, D. M. (1982). Probabilistic reasoning in clinical medicine: Problems and opportunities. In D.Kahneman, P. Slovic, \& A. Tversky (Eds.), Judgment under uncertainty: Heuristics and biases (pp. 249-267). Cambridge: Cambridge University Press.

Einhorn, H. J. \& Hogarth, R. M. (1982). Prediction, diagnosis and causal thinking in forecasting. Journal of forecasting, 1, 1-14. 
Einhorn, H. J. \& Hogarth, R. M. (1983). A theory of diagnostic inference: Studying causality (Rep. No. 4). Chicago: University of Chicago Business School, Centre for Decision Research.

Ennett, C. M. (2003). Imputation of missing values by integrating artificial neural networks and case-based reasoning. PhD thesis,, Ottawa, ON, 2003. Dept of Systems and Computer Engineering, Carleton University.

Fischhof, B. (1977). Perceived informativeness of facts. Journal of Experimental Psychology: Human Perception and Performance, 3, 349-358.

Fischhof, B. (1982). For those condemned to study the past. In D.Kahneman, P. Slovic, \& A. Tversky (Eds.), Judgment under uncertainty: heuristics and biases (pp. 335354). Cambridge: Cambridge University Press.

Fischhof, B., Slovic, P., \& Linchtenstein, S. (1977). Knowing with certainty: The appropriateness of extreme confidence. Journal of Experimental Psychology: Human Perception and Performance, 3, 552-564.

Friedman, C. P., Elstein, A. S., Wolf, F. M., Murphy, G. C., Franz, T. M., Heckerling, P. S. et al. (1999). Enhancement of Clinicians' Diagnostic Reasoning by ComputerBased Consultation. Journal of American Medical Association, 282, 1851-1856.

Frize, M. \& Walker, C. R. (2000). Clinical Decision-Support Systems for Intensive Care Units Using Case-Based Reasoning. Medical Engineering and Physics, 22, 671677. 
Gigerenzer, G. \& Todd, P. M. (1999). Simple Heuristics That Make Us Smart. New York: Oxford University Press, Inc.

Gilovich, T., Griffin, D. W., \& Kahneman, D. (2002). Heuristics and biases: the psychology of intuitive judgement. Cambridge, U.K. ; New York: Cambridge University Press.

Goldberg, L. R. (1968). Simple models or simple processes? Some research on clinical judgments. American Psychologist, 23, 422-432.

Hanley, J. A. (1989). Receiver Operating Characteristic (ROC) Methodology: The State of the Art. Critical Reviews in Diagnostic Imaging, 29, 307-335.

Hanley, J. A. \& McNeil, B. J. (1982). The Meaning and Use of the Area under a Receiver Operating Characteristic (ROC) Curve. Radiology, 143, 29-36.

Horbar, J. D. \& Leahy, K. A. (1995). An assessment of data quality in the Vermont-Oxford Trials Network database. Controlled Clinical Trials, 6, 51-61.

Hosmer, D. W. \& Lemshow, S. (2000). Applied Logistic Regression. (2 ed.) New York: John Wiley \& Sons, Inc.

Johnston, M. E., Langton, K. B., Haynes, R. B., \& Mathieu A. (1994). Effects of Computer-based Clinical Decision Support Systems on clinician Performance and Patient Outcome. A critical Appraisal of Research. Ann Intern Med, 120, 135-42.

Kahneman, D. (1973). Attention and effort. Englewood Cliffs, N.J: Prentice-Hall. 
Klatzky, R. L., Geiwitz, J., \& Fischer, S. C. (1994). Using statistics in clinical practice. In M.S.Bogner (Ed.), Human Error in Medicine (pp. 123-140). Hillsdale, New Jersey: Lawrence Erlbaum Associates, Inc.

Klaus-Dieter, a., Bergmann, R., Wess, S., Manago, M., Auriol, E., Larichev, O. I. et al. (1998). Case-based reasoning for medical decision support tasks: The Inreca approach. Artificial Intelligence in Medicine 12 (1998), 12, 25-41.

Klein, G. (1998). Sources of power:how people make decisions. Cambridge, Mass: MIT Press.

Lisboa, P. J. G. (2002). A review of evidence of health benefit from artificial neural networks in medical intervention. Neural Networks, 15, 11-39.

McFall, R. M. \& Treat, T. A. (1999). Quantifying the information value of clinical assessments with signal detection theory. Annual Review of Psychology, 50, 215241.

Meehl, P. E. (1954). Clinical versus statistical prediction: a theoretical analysis and a review of the evidence. --. Minneapolis: University of Minnesota Press.

Menard, S. (2002). Applied Logistic Regression Analysis. (vols. 106) Thousand Oaks: Sage Plublications, Inc.

Mynatt, C. R., Doherty, M. E., \& Tweney, R. D. (1977). Confirmation bias in a simulated research environment: An experimental study of scientific inference. Quarterly Journal of Experimental Psychology, 29, 85-95. 
Oskamp, S. (1965). Overconfidence in case-study judgments. Journal of Consulting Psychology, 29, 261-265.

Pampel, F. C. (2000). Logistic Regression, A Primer. (vols. 132) Thousand Oaks: Sage Publications, Inc.

Pedhazur, E. J. (1997). Prediction. In Multiple Regression in Behavioral Research, Explanation and Prediction (Third ed., pp. 195-240). Orlando: Harcourt, Inc.

Plous, S. (1993). The Psychology of Judgment And Decision Making. McGraw-Hill.

Richardson, D. K., Corcoran, J. D., Escabar, G. J., \& Lee, S. K. (2001). SNAP-II and SNAPPE-II: Simplified newborn illness severity and mortality risk scores. The Journal of Pediatrics, 138, 93-100.

Richardson, D. K., Gray, J. E., McCormick, M. C., Workman, K., \& Goldmann, D. A. (1993). Score for Neonatal Acute Physiology: A physiologic Severity Index for Neonatal Intensive Care. Pediatrics, 91.

Savage, L. J. (1954). The foundations of statistics. New York: Wiley.

Schmidt, R., Montani, S., Bellazi, R., Portinale, L., \& Gierl, L. (2001). Case-Based Reasoning for medical knowledge-based systems. International Journal of Medical Informatics, 64, 355-367. 
Schoen, C. D., Osborn, R., \& Blendon, R. (2000). Commonwealth Fund 2000 international health policy survey of physicians' perspectives on quality. New York: Commonwealth Fund.

Schwartz, S. \& Griffin, T. (1986). Medical Thinking. Springer-Verlag.

Schwartz, W. B., Kassirer, J. P., \& Essig, A. (1973). Decision analysis and clinical judgment. The American Journal of Medicine, 55, 459-472.

Seitz, A., Uhrmacher, A. M., \& Damm, D. (1999). "Case-based prediction in experimental medical studies"Seitz A.Uhrmacher A.M.,Damm D. Artificial Intelligence in Medicine, 15, 255-273.

Sheridan, T. B. \& Thompson, J. M. (1994). People vs. computers in medicine. In M.S.Bogner (Ed.), Human Error in Medicine (pp. 141-158). Hillsdale, New Jersey: Lawrence Earlbaum Associates, Inc.

Simon, H. A. (1956). Rational choice and the structure of the envrionment. Psychological review, 63, 129-138.

Smith, P. J. \& Geddes, N. G. (2003). A cognitive Systems Engineering Approach to the Design of Decision Support Systems. In J.A.Jacko \& A. Sears (Eds.), The Human Computer Interaction Handbook. Fundamentals, Evolving Technologies and Emerging Applications (pp. 656-676). Mahwah, New Jersey: Lawrence Erlbaum Associates, Inc. 
Sox, H. C., Blatt, M. A., Higgins, M. C., \& Marton, K. I. (1988). Medical Decision Making. Butterworths-Heinemann Publishing.

Swets, J., Dawes, R. M., \& Monohan, J. (2000). Psychological science can improve diagnostic decisions. Psychological Science in the Public Interest, 1, 1-26.

Szklo, M. \& Nieto, J. F. (2000). Epidemiology: Beyond the Basics. Gaithersburg, Maryland: Aspen Publishers Inc.

Terrin, N., Schmid, C. H., Griffith, J. L., D'Agostino, R. B., \& Selker, H. P. (2003). External validity of predictive models: A comparison of Logistic regression, classification trees, and neural networks. Journal of Clinical Epidemiology, 56, $721-729$.

Tversky, A. \& Kahneman, D. (1973). Availability: A heuristic for judging frequency and probability. Cognitive Psychology, 5, 207-232.

Tversky, A. \& Kahneman, D. (2001). Judgment under uncertainty: heuristics and biases. In D.Kahneman, P. Slovic, \& A. Tversky (Eds.), Judgment Under Uncertainty: Heuristics and biases (Cambridge: Cambridge University Press.

Von Neuman, J. \& Morgenstern, O. (1947). Theory of games and economic behavior. Princeton, NJ: Princeton University Press.

Wager, K. A., Lee, F. W., White, A. W., \& Ward, D. M. (2000). Impact of an electronic medical record system on community-based primary care practices. Journal of the American Board of Family Practice, 13, 338-348. 
Zhang, H. \& Singer, B. (1999). Recursive Partitioning in the Health Sciences. New York, NY: Springer-Verlag Inc. 